\title{
PARTIALLY CONDENSING MAPPINGS IN PARTIALLY ORDERED NORMED LINEAR SPACES AND APPLICATIONS TO FUNCTIONAL INTEGRAL EQUATIONS
}

\author{
BAPURAO C. DHAGE
}

\begin{abstract}
In this paper, the author introduces a notion of partially condensing mappings in a partially ordered normed linear space and proves some hybrid fixed point theorems under certain mixed conditions of algebra, analysis and topology. The applications of abstract results presented here are given to some nonlinear functional integral equations for proving the existence as well as global attractivity of the comparable solutions under certain monotonicity conditions. The abstract theory presented here is very much useful to develop the algorithms for the solutions of some nonlinear problems of analysis and allied areas of mathematics. A realization of of our hypotheses is also indicated by a numerical example.
\end{abstract}

\section{Introduction}

It is well-known that compact sets are next to the finite sets useful for handling in the mathematical formulations and so, several results from analysis and topology use compactness to derive some far reaching conclusions. The topological methods or fixed point theorems involving the compactness arguments are useful only to prove existence of the solutions of some nonlinear equations and do not give any computational scheme or algorithm for solving such problems. However if we combine the compactness or its generalizations in terms of measure of noncompactness with some algebraic arguments, then it is possible to develop the algorithms for the solutions of nonlinear problems under some suitable conditions. The work along this line is of great interest and which is the main motivation of the present paper. Here, we combine the topological arguments with some order related hypotheses to prove some hybrid fixed point theorems for the mappings in ordered spaces and apply newly developed abstract result to obtain a algorithm for the solutions of a functional integral equation under some mixed compactness and monotonic conditions. We claim that the results of this paper are new to the literature.

Received October 7, 2013, accepted Febuary 27, 2014. 2010 Mathematics Subject Classification. 45G10, 45M99, 47H09, 47H10.

Key words and phrases. Partially measure of noncompactness, partially condensing mappings, fixed points, functional integral equation, Existence theorem etc. 
Many attempts have been made in the literature to measure numerically the degree of noncompactness of sets in a normed linear space in terms of certain characteristics of the compactness property. The more is the value of this characteristic the lesser is the compactness and vice versa. Two such Kuratowskii [18] and Hausdorff [15] measures of noncompactness in a metric space are well-known in the literature. However, axiomatic way of approach of the measures of noncompactness is sometimes useful in the study of various qualitative properties of the dynamical systems in nonlinear analysis (cf. [1] and [2]). In this article, we follow the axiomatic way of approach to define a new partially measure of noncompactness in a partially ordered normed linear space and which is subsequently exploited to derive some interesting consequences. We prove some new fixed point theorems (FPTs) for partially condensing mappings in a partially ordered complete normed linear space and apply our abstract results to a certain nonlinear hybrid functional integral equation for proving the existence as well as global attractivity results on unbounded intervals of real line. The abstract theory presented is useful to develop the algorithms for the solutions of some nonlinear problems of analysis and allied areas of mathematics.

\section{Partially ordered linear spaces}

Let $E$ be a real vector or linear space. We introduce a partial order $\leq$ in $E$ as follows. A relation $\preceq$ in $E$ is said to be partial order if it satisfies the following properties: Let $a, b, c, d \in E$ and $\lambda \in \mathbb{R}$.

1. Reflexivity: $a \leq a$ for all $a \in E$,

2. Antisymmetry: $a \preceq b$ and $b \preceq a$ implies $a=b$,

3. Transitivity: $a \leq b$ and $b \leq c$ implies $a \leq c$, and

4. Order linearity: $a \leq b$ and $c \leq d \Longrightarrow a+c \leq b+d$;

and $a \leq b \Longrightarrow \lambda a \leq \lambda b$ for $\lambda \geq 0$.

The linear space $E$ together with a partial order $\leq$ becomes a partially ordered linear or vector space. Two elements $x$ and $y$ in a partially ordered linear space $E$ are called comparable if either the relation $x \leq y$ or $y \leq x$ holds. We introduce a norm $\|\cdot\|$ in a partially ordered linear space $E$ so that $E$ becomes now a partially ordered normed linear space. If $E$ is complete with respect to the metric $d$ defined through the above norm, then it is called a partially ordered complete normed linear space. We frequently need the concept of regulatory of $E$ in what follows. It is known that $E$ is regular if $\left\{x_{n}\right\}$ is a nondecreasing (resp. nonincreasing) sequence in $E$ such that $x_{n} \rightarrow x^{*}$ as $n \rightarrow \infty$, then $x_{n} \leq x^{*}$ (resp. $x_{n} \geq x^{*}$ ) for all $n \in \mathbb{N}$. A few details of an ordered Banach spaces and operator theoretic techniques are given in a series of papers 
Dhage [6, 7, 8, 9], Dhage and Dhage[11] and Dhage et. al [12]. The applications to nonlinear differential and integral may be found in Dhage [7], Dhage and Dhage [10], Dhage et. al [13], Heikkilä and Lakshmikantham [16] and Carl and Hekkilä [5] and the references therein.

Definition 2.1. A mapping $\mathscr{T}: X \rightarrow X$ is called monotone nondecreasing if it preserves the order relation $\leq$, that is, if $x \leq y$ implies $\mathscr{T} x \leq \mathscr{T} y$ for all $x, y \in X$. Similarly, $\mathscr{T}$ is called monotone nonincreasing if $x \leq y$ implies $\mathscr{T} x \geq \mathscr{T} y$ for all $x, y \in X$. A monotone mapping $\mathscr{T}$ is one which is either monotone nondecreasing or monotone nonincreasing on $X$.

The following terminologies may be found in any book on nonlinear operators, equations and applications. See Banas and Goebel [2], Krasnoselskii [17] and the references therein.

An operator $\mathscr{T}$ on a normed linear space $E$ into itself is called compact if $\mathscr{T}(E)$ is a relatively compact subset of $E . \mathscr{T}$ is called totally bounded if for any bounded subset $S$ of $E, \mathscr{T}(S)$ is a relatively compact subset of $E$. If $\mathscr{T}$ is continuous and totally bounded, then it is called completely continuous on $E$.

The following definitions have been introduced in Dhage [8] and are frequently used in the subsequent part of this paper.

Definition 2.2 (Dhage [9]). A mapping $\mathscr{T}: E \rightarrow E$ is called partially continuous at a point $a \in E$ if for $\epsilon>0$ there exists a $\delta>0$ such that $\|\mathscr{T} x-\mathscr{T} a\|<\epsilon$ whenever $x$ is comparable to $a$ and $\|x-a\|<\delta . \mathscr{T}$ called partially continuous on $E$ if it is partially continuous at every point of it. It is clear that if $\mathscr{T}$ is partially continuous on $E$, then it is continuous on every chain $C$ contained in $E$.

Definition 2.3. A mapping $\mathscr{T}: E \rightarrow E$ is called partially bounded if $T(C)$ is bounded for every chain $C$ in $E . \mathscr{T}$ is called uniformly partially bounded if all chains $\mathscr{T}(C)$ in $E$ are bounded by a unique constant. $\mathscr{T}$ is called bounded if $T(E)$ is a bounded subset of $E$.

Definition 2.4. A mapping $\mathscr{T}: E \rightarrow E$ is called partially compact if $\mathscr{T}(C)$ is a relatively compact subset of $E$ for all totally ordered sets or chains $C$ in $E$. $\mathscr{T}$ is called uniformly partially compact if $\mathscr{T}(C)$ is a uniformly partially bounded and partially compact on $E$. $\mathscr{T}$ is called partially totally bounded if for any totally ordered and bounded subset $C$ of $E, \mathscr{T}(C)$ is a relatively compact subset of $E$. If $\mathscr{T}$ is partially continuous and partially totally bounded, then it is called partially completely continuous on $E$.

Remark 2.1. Note that every compact mapping on a partially normed linear space is partially compact and every partially compact mapping is partially totally bounded, however the reverse implications do not hold. Again, every completely continuous mapping is partially completely continuous and every partially completely continuous mapping is partially continuous and partially totally bounded, but the converse may not be true. 
Definition 2.5 (Dhage [8]). The order relation $\preceq$ and the metric $d$ on a non-empty set $E$ are said to be compatible if $\left\{x_{n}\right\}$ is a monotone, that is, monotone nondecreasing or monotone nondecreasing sequence in $E$ and if a subsequence $\left\{x_{n_{k}}\right\}$ of $\left\{x_{n}\right\}$ converges to $x^{*}$ implies that the whole sequence $\left\{x_{n}\right\}$ converges to $x^{*}$. Similarly, given a partially ordered normed linear space $(E, \preceq,\|\cdot\|)$, the order relation $\leq$ and the norm $\|\cdot\|$ are said to be compatible if $\leq$ and the metric $d$ defined through the norm $\|\cdot\|$ are compatible.

Clearly, the set $\mathbb{R}$ of real numbers with usual order relation $\leq$ and the norm defined by the absolute value function has this property. Similarly, every partially compact subset of the space $C(J, \mathbb{R})$ with usual order relation defined by $x \leq y$ if and only if $x(t) \leq y(t)$ for all $t \in J$ and the usual standard supremum norm $\|\cdot\|$ defined by $\|x\|=\sup _{t \in J}|x(t)|$ are compatible.

The following applicable hybrid fixed point theorem is proved in Dhage [8] .

Theorem 2.1. Let E be a partially ordered linear space and suppose that there is a norm in $E$ such that $E$ is a normed linear space. Let $\mathscr{T}: E \rightarrow E$ be a nondecreasing, partially compact and continuous mapping. Further if the order relation $\leq$ and the norm $\|\cdot\|$ in $E$ are compatible and if there is an element $x_{0} \in E$ satisfying $x_{0} \leq \mathscr{T} x_{0}$ or $x_{0} \geq \mathscr{T} x_{0}$, then $\mathscr{T}$ has a fixed point $x^{*}$ and the sequence $\left\{T^{n} x_{0}\right\}$ of successive iterations converges to $x^{*}$.

We note that Theorem 2.1 is very much useful for proving the existence theorems for several dynamical systems in nonlinear analysis modeled on nonlinear differential and integral equations. Here, in the following section we generalize above fixed point theorem under weaker conditions via measure of noncompactness and apply it to obtain existence of the solutions of a certain nonlinear functional integral equation in a constructive way.

\section{Partially condensing mappings}

At the beginning of this section, we present some basic facts concerning the partially measures of noncompactness in a partially ordered normed linear space. Assume that $(E, \|$. II) is an infinite dimensional partially ordered Banach space with zero element $\theta$. If $C$ is a chain in $E$, then $C^{\prime}$ denotes the set of all limit points of $C$ in $E$. The symbol $\bar{C}$ stands for the closure of $C$ in $E$ defined by $\bar{C}=C \cup C^{\prime}$. Clearly, $\inf C$, sup $C \in \bar{C}$ provided $\inf C$ and $\sup C$ exist. The $\sup C$ is an element $z \in E$ such that for every $\epsilon>0$ there exists a $c \in C$ such that $d(c, z)<\epsilon$ and $x \leq z$ for all $x \in C$. Similarly, $\inf C$ is defined in the same way. Then $\bar{C}$ is again a chain, called the closed chain in $E$. Thus, $\bar{C}$ is the intersection of all closed chains containing $C$. In what follows, we denote by $\mathscr{P}_{c l}(E), \mathscr{P}_{b d}(E), \mathscr{P}_{r c p}(E), \mathscr{P}_{c h}(E), \mathscr{P}_{b d, c h}(E), \mathscr{P}_{r c p, c h}(E)$ the family of all nonempty and closed, bounded, relatively compact, chains, bounded chains and relatively compact chains of $E$ respectively. Now we introduce the concept of partially measure of noncompactness in $E$ on the lines of usual classical theory. 
Definition 3.1. A mapping $\mu^{p}: \mathscr{P}_{b d, c h}(E) \rightarrow \mathbb{R}_{+}=[0, \infty)$ is said to be a partially measure of noncompactness in $E$ if it satisfies the following conditions:

$1^{o} \varnothing \neq\left(\mu^{p}\right)^{-1}(\{0\}) \subset \mathscr{P}_{r c p, c h}(E)$,

$2^{o} \mu^{p}(\bar{C})=\mu^{p}(C)$,

$3^{o} \mu^{p}$ is nondecreasing, i.e., if $C_{1} \subset C_{2} \Rightarrow \mu^{p}\left(C_{1}\right) \leq \mu^{p}\left(C_{2}\right)$, and

$4^{o}$ If $\left\{C_{n}\right\}$ is a sequence of closed chains from $\mathscr{P}_{b d, c h}(E)$ such that $C_{n+1} \subset C_{n},(n=1,2, \ldots)$ and if $\lim _{n \rightarrow \infty} \mu^{p}\left(C_{n}\right)=0$, then the intersection set $\bar{C}_{\infty}=\bigcap_{n=1}^{\infty} C_{n}$ is nonempty.

The family of sets described in $1^{o}$ is said to be the kernel of the measure of noncompactness $\mu^{p}$ and is defined as

$$
\operatorname{ker} \mu^{p}=\left\{C \in \mathscr{P}_{b d, c h}(E) \mid \mu^{p}(C)=0\right\} .
$$

Clearly, $\operatorname{ker} \mu^{p} \subset \mathscr{P}_{r c p, c h}(E)$. Observe that the intersection set $C_{\infty}$ from condition $4^{o}$ is a member of the family $\operatorname{ker} \mu^{p}$. In fact, since $\mu^{p}\left(C_{\infty}\right) \leq \mu^{p}\left(C_{n}\right)$ for any $n$, we infer that $\mu^{p}\left(C_{\infty}\right)=0$. This yields that $C_{\infty} \in \operatorname{ker} \mu^{p}$. This simple observation will be essential in our further investigations.

Te partially measure $\mu^{p}$ of noncompactness is called sublinear if it satisfies

$5^{o} \mu^{p}\left(C_{1}+C_{2}\right) \leq \mu^{p}\left(C_{1}\right)+\mu^{p}\left(C_{2}\right)$ for all $C_{1}, C_{2} \in \mathscr{P}_{b d, c h}(E)$ and

$6^{o} \mu^{p}(\lambda C)=|\lambda| \mu^{p}(C)$ for $\lambda \in \mathbb{R}$.

Again, $\mu^{p}$ is said to satisfy maximum property if

$7^{o} \mu^{p}\left(C_{1} \cup C_{2}\right)=\max \left\{\mu^{p}\left(C_{1}\right), \mu^{p}\left(C_{2}\right)\right\}$.

Finally, $\mu^{p}$ is said to be full or complete if

$8^{o} \operatorname{ker} \mu^{p}=\mathscr{P}_{r c p, c h}(E)$.

Example 3.1. Define two functions $\alpha^{p}, \beta^{p}: \mathscr{P}_{b d, c h}(E) \rightarrow \mathbb{R}_{+}$by

$$
\alpha^{p}(C)=\inf \left\{r>0 \mid C=\bigcup_{i=1}^{n} C_{i}, \operatorname{diam}\left(C_{i}\right) \leq r \forall i\right\}
$$

where, $C \in \mathscr{P}_{b d, c h}(E)$ and $\operatorname{diam}\left(C_{i}\right)=\sup \left\{\|x-y\|: x, y \in C_{i}\right\}$, and

$$
\beta^{p}(C)=\inf \left\{r>0 \mid C \subset \bigcup_{i=1}^{n} \mathscr{B}\left(x_{i}, r\right) \text { for some } x_{i} \in E\right\}
$$

where, $\mathscr{B}\left(x_{i}, r\right)=\left\{x \in E:\left\|x_{i}-x\right\|<r\right\}$. It is easy to prove that $\alpha^{p}$ and $\beta^{p}$ are partially measures of noncompactness called respectively the partially Kuratowskii and partially ball or Hausdorff measures of noncompactness in $E$. 
The above two partially Kuratowskii and Hausdorff measures of noncompactness $\alpha^{p}$ and $\beta^{p}$ are sublinear, full and enjoy the maximum property in $E$. The verification of this claim is same as classical Kuratowskii and Hausdorff measures of noncompactness and so we omit the details.

Definition 3.2. A mapping $\psi: \mathbb{R}_{+} \rightarrow \mathbb{R}_{+}$is called a dominating function or, in short, $\mathscr{D}$-function if it is an upper semi-continuous and monotonic nondecreasing function satisfying $\psi(0)=0$.

There do exist $\mathscr{D}$-functions and commonly used $\mathscr{D}$-functions are

$$
\begin{aligned}
& \psi(r)=k r, \text { for some constant } k>0, \\
& \psi(r)=\frac{L r}{K+r}, \text { for some constants } L>0, K>0, \\
& \psi(r)=\tan ^{-1} r, \\
& \psi(r)=\log (1+r), \\
& \psi(r)=e^{r}-1 .
\end{aligned}
$$

The above defined $\mathscr{D}$-functions have been widely used in the existence theory of nonlinear differential and integral equations.

Remark 3.1. If $\phi, \psi \mathbb{R}_{+} \rightarrow \mathbb{R}_{+}$are two $\mathscr{D}$-functions, then i) $\phi+\psi$, ii) $\lambda \phi, \lambda>0$, and iii) $\phi \circ \psi$ are also $\mathscr{D}$-functions on $\mathbb{R}_{+}$. The class of $\mathscr{D}$-functions on $\mathbb{R}_{+}$is denoted by $\mathfrak{D}$.

Definition 3.3. A nondecreasing mapping $\mathscr{T}: E \rightarrow E$ is called partially nonlinear $\mathscr{D}$-setLipschitz if there exists a $\mathscr{D}$-function $\psi$ such that

$$
\mu^{p}(\mathscr{T} C) \leq \psi\left(\mu^{p}(C)\right)
$$

for all bounded chain $C$ in $E$. $\mathscr{T}$ is called partially $k$-set-Lipschitz if $\mu^{p}(r)=k r, k>0$. $\mathscr{T}$ is called partially $k$-set-contraction if it is a partially $k$-set-Lipschitz with $k<1$. Finally, $\mathscr{T}$ is called a partially nonlinear $\mathscr{D}$-set-contraction if it is a partially nonlinear $\mathscr{D}$-Lipschitz with $\mu^{p}(r)<r$ for $r>0$.

Remark 3.2. It is clear tat every partially $k$-set-contraction is a partially nonlinear $\mathscr{D}$-setcontraction an every partially nonlinear $\mathscr{D}$-set-contraction is partially condensing, however, the converse implications may not be true. Actually, it is very difficult to prove practically a selfmaping of a normed linear space is partially condensing and we rarely come across a mapping of this kind. But the mappings with nonlinear $\mathscr{D}$-set-contraction and $k$ - $\mathscr{D}$-setcontraction are available in the literature.

The following lemma (see Dhage [8, page 159]) is frequently used in the analytical fixed point theory of metric spaces. 
Lemma 3.1 (Dhage [6]). Let $\psi: \mathbb{R}_{+} \rightarrow \mathbb{R}_{+}$be a $\mathscr{D}$-function satisfying $\psi(r)<r$ for $r>0$. Then $\lim _{n \rightarrow \infty} \psi^{n}(t)=0$ for each $t \in \mathbb{R}_{+}$and vice-versa.

Our main result of this section is as follows.

Theorem 3.1. Let $(E, \preceq,\|\cdot\|)$ be a regular partially ordered complete normed linear space such that the order relation $\leq$ and the norm $\|\cdot\|$ are compatible. Let $\mathscr{T}: E \rightarrow E$ be a nondecreasing, partially continuous and partially bounded mapping. If $T$ is partially nonlinear $\mathscr{D}$-setcontraction and if there exists an element $x_{0} \in E$ such that $x_{0} \leq \mathscr{T} x_{0}$, then $\mathscr{T}$ has a fixed point $x^{*}$ and the sequence $\left\{\mathscr{T}^{n} x_{0}\right\}$ of successive iterations converges monotonically to $x^{*}$.

Proof. Define a sequence $\left\{x_{n}\right\}$ of points in $E$ by

$$
x_{n+1}=\mathscr{T} x_{n}, \quad n=0,1,2, \ldots
$$

Since $\mathscr{T}$ is nondecreasing and $x_{0} \preceq \mathscr{T} x_{0}$, we have that

$$
x_{0} \leq x_{1} \leq x_{2} \leq \cdots \leq x_{n} \leq \cdots
$$

Denote

$$
C_{n}=\left\{x_{n}, x_{n+1}, \ldots\right\}
$$

for $n=0,1,2, \ldots$. By construction, each $C_{n}$ is a bounded and closed chain in $E$ and

$$
C_{n}=T\left(C_{n-1}\right), \quad n=0,1,2, \ldots
$$

Moreover,

$$
C_{0} \supset C_{1} \supset \cdots \supset C_{n} \supset \cdots .
$$

Therefore, by nondecreasing nature of $\mu^{p}$ we obtain

$$
\begin{aligned}
\mu^{p}\left(C_{n}\right) & =\mu^{p}\left(\mathscr{T}\left(C_{n-1}\right)\right) \\
& \leq \psi\left(\mu^{p}\left(C_{n-1}\right)\right) \\
& \leq \psi^{2}\left(\mu^{p}\left(C_{n-2}\right)\right) \\
& \vdots \\
& \leq \psi^{n}\left(\mu^{p}\left(C_{0}\right)\right)
\end{aligned}
$$

Taking the limit superior as $n \rightarrow \infty$ in the above equality (3.4), in view of Lemma 3.1 we obtain that

$$
\lim _{n \rightarrow \infty} \mu^{p}\left(C_{n}\right) \leq \limsup _{n \rightarrow \infty} \psi^{n}\left(\mu^{p}\left(C_{0}\right)\right)=\lim _{n \rightarrow \infty} \psi^{n}\left(\mu^{p}\left(C_{0}\right)\right)=0
$$


Hence, by condition $\left(4^{o}\right)$ of $\mu^{p}$,

$$
\bar{C}_{\infty}=\bigcap_{n=1}^{\infty} C_{n} \neq \varnothing \quad \text { and } \quad C_{\infty} \in \mathscr{P}_{r c p, c h}(E) .
$$

From (3.5) it follows that for every $\epsilon>0$ there exists an $n_{0} \in \mathbb{N}$ such that

$$
\mu^{p}\left(C_{n}\right)<\epsilon \quad \forall n \geq n_{0} .
$$

This shows that $\bar{C}_{n_{0}}$ and consequently $\bar{C}_{0}$ is a compact chain in $E$. Hence, $\left\{x_{n}\right\}$ has a convergent subsequence. Further since the order relation $\leq$ and the norm $\|\cdot\|$ are compatible, the whole sequence $\left\{x_{n}\right\}=\left\{\mathscr{T}^{n} x_{0}\right\}$ is convergent and converges monotonically to a point, say $x^{*} \in \bar{C}_{0}$. Since the ordered space space $E$ is regular, we have that $x_{n} \leq x^{*}$. Finally, from the partial continuity of $\mathscr{T}$, we get

$$
\mathscr{T} x^{*}=\mathscr{T}\left(\lim _{n \rightarrow \infty} x_{n}\right)=\lim _{n \rightarrow \infty} \mathscr{T} x_{n}=\lim _{n \rightarrow \infty} x_{n+1}=x^{*} .
$$

This completes the proof.

Theorem 3.2. Let $(E, \preceq,\|\cdot\|)$ be a regular partially ordered complete normed linear space such that the order relation $\leq$ and the norm $\|\cdot\|$ are compatible. Let $\mathscr{T}: E \rightarrow E$ be a nondecreasing, partially continuous and partially bounded mapping. If $T$ is partially nonlinear $\mathscr{D}$-setcontraction and if there exists an element $x_{0} \in E$ such that $x_{0} \leq \mathscr{T} x_{0}$, then $\mathscr{T}$ has a fixed point $x^{*}$ and the sequence $\left\{\mathscr{T}^{n} x_{0}\right\}$ of successive iterations converges monotonically to $x^{*}$.

Proof. The proof is similar to Theorem 3.1 and hence we omit the details.

Remark 3.3. If the set $S_{\mathscr{T}}$ of solutions to the operator equation $T x=x$ is a chain, then all the solutions belonging to $E$ are comparable. Further, if $\mu^{p}(S \mathscr{T})>0$, then $\mu^{p}\left(S_{\mathscr{T}}\right)=\mu^{p}\left(\mathscr{T} S_{\mathscr{T}}\right) \leq$ $\psi\left(\mu^{p}\left(S_{\mathscr{T}}\right)\right)<\mu^{p}\left(S_{\mathscr{T}}\right)$ which is a contradiction. Consequently, $S_{\mathscr{T}} \in \operatorname{ker} \mu^{p}$. This simple fact has been utilized in the study of qualitative properties of the dynamic systems under consideration.

Remark 3.4. The regularity of the partially ordered normed linear space $E$ in above Theorems 3.1 and 3.2 may be replaced with a continuity condition which is stronger than partial continuity of the operators $\mathscr{T}$ on $E$.

Remark 3.5. Suppose that the order relation $\leq$ is introduced in $E$ with the help of an order cone $\mathscr{K}$ which is a non-empty closed set $\mathscr{K}$ in $E$ satisfying (i) $\mathscr{K}+\mathscr{K} \subseteq \mathscr{K}$, (ii) $\lambda \mathscr{K} \subseteq \mathscr{K}$ and (iii) $\{-\mathscr{K}\} \cap \mathscr{K}=\{0\}$ (cf. [16]). Then the order relation $\preceq$ in $E$ is defined as $x \preceq y \Longleftrightarrow y-x \in \mathscr{K}$. The element $x_{0} \in E$ satisfying $x_{0} \leq \mathscr{T} x_{0}$ in above Theorem 3.1 is called a lower solution of the operator equation $x=\mathscr{T} x$. If the operator equation $x=\mathscr{T} x$ has more than one lower solution 
and set of all these lower solutions are comparable, then the corresponding set $S$ of solutions to above operator equation is a chain and hence all solutions in $S$ are comparable. To see this, let $x_{0}$ and $y_{0}$ be any two lower solutions of the above operator equation such that $x_{0} \leq y_{0}$ and let $x^{*}$ and $y^{*}$ respectively be the corresponding solutions under the conditions of Theorem 3.1. Now, by definition of $\leq$, one has $y_{0}-x_{0} \in \mathscr{K}$ and from monotone nondecreasing nature of $T$ it follows that $T^{n} y_{0}-T^{n} x_{0} \in \mathscr{K}$. Since $\mathscr{K}$ is closed, we have that $y^{*}-x^{*} \in \mathscr{K}$ or $x^{*} \leq y^{*}$.

Theorem 3.3. Let $(E, \preceq,\|\cdot\|)$ be a regular partially ordered complete normed linear space such that the order relation $\leq$ and the norm $\|\cdot\|$ are compatible. Suppose that $\mathscr{T}: E \rightarrow E$ is a partially continuous, nondecreasing and partially nonlinear $k$-set-contraction with $k<1$. If $\mathscr{T}$ is partially bounded and there exists an element $x_{0} \in E$ such that $x_{0} \leq \mathscr{T} x_{0}$ or $x_{0} \succeq \mathscr{T} x_{0}$, then $\mathscr{T}$ has a fixed point $x^{*}$ and the sequence $\left\{\mathscr{T}^{n} x_{0}\right\}$ of successive iterations converges monotonically to $x^{*}$.

Proof. The proof is similar to Theorem 3.1 and so we omit the details.

Corollary 3.1 (Dhage [8]). Let $(E, \preceq,\|\cdot\|)$ be a regular partially ordered complete normed linear space such that the order relation $\leq$ and the norm $\|\cdot\|$ are compatible. Suppose that $\mathscr{T}: E \rightarrow E$ is a partially continuous, nondecreasing and partially compact mapping. If $\mathscr{T}$ is partially bounded and there exists an element $x_{0} \in E$ such that $x_{0} \leq \mathscr{T} x_{0}$ or $x_{0} \geq T x_{0}$, then $\mathscr{T}$ has a fixed point $x^{*}$ and the sequence $\left\{\mathscr{T}^{n} x_{0}\right\}$ of successive iterations converges monotonically to $x^{*}$.

Before giving a further generalization of Theorems 3.1 and 3.2, we state a useful definition.

Definition 3.4. A nondecreasing mapping $\mathscr{T}: E \rightarrow E$ is called partially condensing if for any bounded chain $C$ in $E, \mu^{p}(\mathscr{T}(C))<\mu^{p}(C)$ for $\mu^{p}(C)>0$.

We remark that every partially compact and partially nonlinear $\mathscr{D}$-set-contraction mappings are partially condensing, however the reverse implications may not hold.

Theorem 3.4. Let $(E, \preceq,\|\cdot\|)$ be a regular partially ordered complete normed linear space such that the order relation $\leq$ and the norm $\|\cdot\|$ are compatible. Suppose that $\mathscr{T}: E \rightarrow E$ is a partially continuous, nondecreasing and partially condensing mapping. If $\mathscr{T}$ is partially bounded and there exists an element $x_{0} \in E$ such hat $x_{0} \leq \mathscr{T} x_{0}$ or $x_{0} \geq \mathscr{T} x_{0}$, then $\mathscr{T}$ has a fixed point $x^{*}$ and the sequence $\left\{\mathscr{T}^{n} x_{0}\right\}$ of successive iterations converges monotonically to $x^{*}$.

Proof. The proof is standard and hence we omit the details.

Remark 3.6. We note that the proofs of Theorems 3.1 and 3.2 do not make any use of the linear structure of the underlined space $E$, and therefore, Theorems 3.1, 3.2, 3.3 and 3.4 also remain true in the setting of a partially ordered metric space $E$. 
In view of above Remark 3.6, a slight generalization of Theorems 3.1 3.2, 3.3 and 3.4 are as follows.

Theorem 3.5. Let $(E, \preceq, d)$ be a regular partially ordered complete metric space such that the order relation $\leq$ and the metric d are compatible. Let $\mathscr{T}: E \rightarrow E$ be a partially continuous, nondecreasing and partially condensing mapping. If $\mathscr{T}$ is partially bounded and there exists an element $x_{0} \in E$ such hat $x_{0} \leq \mathscr{T} x_{0}$ or $x_{0} \geq \mathscr{T} x_{0}$, then $\mathscr{T}$ has a fixed point $x^{*}$ and the sequence $\left\{\mathscr{T}^{n} x_{0}\right\}$ of successive iterations converges monotonically to $x^{*}$.

Corollary 3.2. Let $(E, \preceq, d)$ be a regular partially ordered complete metric space such that the order relation $\leq$ and the norm $d$ are compatible. Suppose that $\mathscr{T}: E \rightarrow E$ is a nondecreasing, partially continuous and partially bounded mapping. If $T$ is partially nonlinear $\mathscr{D}$-setcontraction and if there exists an element $x_{0} \in E$ such that $x_{0} \leq \mathscr{T} x_{0}$, then $\mathscr{T}$ has a fixed point $x^{*}$ and the sequence $\left\{\mathscr{T}^{n} x_{0}\right\}$ of successive iterations converges monotonically to $x^{*}$.

Corollary 3.3. Let $(E, \preceq, d)$ be a regular partially ordered complete metric space such that the order relation $\leq$ and the norm d are compatible. Suppose that $\mathscr{T}: E \rightarrow E$ is a partially continuous, nondecreasing and partially $k$-set-contraction with $k<1$. If $\mathscr{T}$ is partially bounded and there exists an element $x_{0} \in E$ such hat $x_{0} \leq \mathscr{T} x_{0}$ or $x_{0} \geq T x_{0}$, then $\mathscr{T}$ has a fixed point $x^{*}$ and the sequence $\left\{\mathscr{T}^{n} x_{0}\right\}$ of successive iterations converges monotonically to $x^{*}$.

Corollary 3.4. Let $(E, \preceq, d)$ be a regular partially ordered complete metric space such that the order relation $\leq$ and the norm $d$ are compatible. Suppose that $\mathscr{T}: E \rightarrow E$ is a partially continuous, nondecreasing and compact mapping. If $\mathscr{T}$ is partially bounded and there exists an element $x_{0} \in E$ such hat $x_{0} \leq \mathscr{T} x_{0}$ or $x_{0} \geq T x_{0}$, then $\mathscr{T}$ has a fixed point $x^{*}$ and the sequence $\left\{\mathscr{T}^{n} x_{0}\right\}$ of successive iterations converges monotonically to $x^{*}$.

Remark 3.7. The regularity of the partially ordered metric space $E$ in above Theorem 3.5 may be replaced with a stronger continuity condition than partial continuity of the mappings $\mathscr{T}$ on $E$. Furthermore, the fixed point set $\mathscr{F}_{\mathscr{T}}$ of comparable elements of the mapping $\mathscr{T}$ in $S$ is a member of $\operatorname{ker} \mu^{p}$.

\section{FPTs of Krasnoselskii and Dhage type}

The study of hybrid fixed point theorems for sum of two operators is attributed to Krasnoselskii [17] whereas the study involving the product of two operators in Banach algebra is attributed to Dhage [7]. Again the study of fixed point theorems in Banach algebras involving the sum as well as product of operators is credited to Dhage [7]. Here, we prove the analogous results for the sum and the product of operators in partially ordered complete normed linear 
spaces which are useful in applications to perturbed nonlinear differential and integral equations for proving the existence and attractivity of solutions under mixed compact, Lipschitz and monotonic conditions.

\subsection{FPTs of Krasnoselskii type}

Definition 4.1. Let $(E, \preceq,\|\cdot\|)$ be a partially ordered normed linear space. A mapping $\mathscr{T}: E \rightarrow$ $E$ is called partially nonlinear $\mathscr{D}$-Lipschitz if there exists a $\mathscr{D}$-function $\psi: \mathbb{R}_{+} \rightarrow \mathbb{R}_{+}$such that

$$
\|\mathscr{T} x-\mathscr{T} y\| \leq \psi(\|x-y\|)
$$

for all comparable elements $x, y \in E$. If $\psi(r)=k r, k>0$, then $\mathscr{T}$ is called a partially Lipschitz with a Lipschitz constant $k$. If $k<1, \mathscr{T}$ is called a partially contraction with contraction constant $k$. Finally, $\mathscr{T}$ is called nonlinear $\mathscr{D}$-contraction if it is a nonlinear $\mathscr{D}$-Lipschitz with $\psi(r)<r$ for $r>0$.

In a recent paper [8], the author proved the following fixed point result in a partially ordered Banach space which combines contraction mapping principle and topological fixed point principle developed in the same paper. Also see Nieto and Lopez [19] and the references cited therein.

Theorem 4.1 (Dhage [8]). Let $(E, \preceq,\|\cdot\|)$ be a partially ordered complete normed linear space such that the order relation $\leq$ and the norm $\|\cdot\|$ in $E$ are compatible. Let $\mathscr{A}, \mathscr{B}: E \rightarrow E$ be two nondecreasing operators such that

(a) $\mathscr{A}$ is continuous and partially nonlinear $\mathscr{D}$-contraction,

(b) $\mathscr{B}$ is continuous and partially compact,

(c) there exists an element $x_{0} \in E$ such that $x_{0} \leq \mathscr{A} x_{0}+\mathscr{B} y$ for all $y \in E$, and

(d) every pair of elements $x, y \in E$ has a lower and an upper bound in $E$.

Then the operator equation

$$
\mathscr{A} x+\mathscr{B} x=x
$$

has a solution in E.

Note that the hypothesis (c) is just more than that of a lower solution of the operator equation (4.1) and the hypothesis (d) holds in particular if $E$ is a lattice. In the following we improve above Theorem 4.1 under weaker conditions (c) and (d) thereof with a different proof. Before going to the main fixed point result we prove a useful lemma which we need in what follows. 
Lemma 4.2. Let $(X, \preceq,\|\cdot\|)$ be a partially ordered complete normed linear space. If $\mathscr{T}: E \rightarrow E$ is a nondecreasing and partially nonlinear $\mathscr{D}$-Lipschitz mapping, then for any bounded chain $C$ in $E$, we have

$$
\alpha^{p}(\mathscr{T} C) \leq \psi\left(\alpha^{p}(C)\right)
$$

where, $\alpha^{p}$ is a partially Kurotowskii measure of noncompactness and $\psi$ is a associated $\mathscr{D}$ function of $\mathscr{T}$ on $E$.

Proof. The proof is similar to standard result for usual nonlinear $\mathscr{D}$-Lipschitz mapping with Kurotowskii measure of noncomactness $\alpha$ in the Banach space $E$. We omit the details.

Theorem 4.2. Let $(E, \preceq,\|\cdot\|)$ be a regular partially ordered complete normed linear space such that the order relation $\leq$ and the norm $\|\cdot\|$ in $E$ are compatible. Let $\mathscr{A}, \mathscr{B}: E \rightarrow E$ be two nondecreasing operators such that

(a) $\mathscr{A}$ is partially bounded and partially nonlinear $\mathscr{D}$-contraction,

(b) $\mathscr{B}$ is partially continuous and partially compact, and

(c) there exists an element $x_{0} \in E$ such that $x_{0} \leq \mathscr{A} x_{0}+\mathscr{B} x_{0}$.

Then the operator equation (4.1) has a solution $x^{*}$ in $E$ and the sequence $\left\{x_{n}\right\}$ of successive iterations defined by $x_{n+1}=\mathscr{A} x_{n}+\mathscr{B} x_{n}, n=0,1, \ldots ;$ converges monotonically to $x^{*}$.

Proof. Define a mapping $\mathscr{T}$ on $E$ by

$$
\mathscr{T} x=\mathscr{A} x+\mathscr{B} x
$$

Since $\mathscr{A}$ and $\mathscr{B}$ are nondecreasing, $\mathscr{T}$ is nondecreasing on $E$. From the partial continuity of the operators $\mathscr{A}$ and $\mathscr{B}$ and the continuity of the binary composition addition, it follows that the operator $\mathscr{T}$ is partially continuous on $E$. As $\mathscr{A}$ is partially bounded and $\mathscr{B}$ is partially continuous and partially compact, we have that $\mathscr{T}$ is a partially bounded operator on $E$. Again, by hypothesis (c), $x_{0} \leq \mathscr{T} x_{0}$. Next, we show that $\mathscr{T}$ is a nonlinear $\mathscr{D}$-set-contraction on $E$. Let $C$ be a bounded chain in $E$. Then by definition of $\mathscr{T}$, we have

$$
\mathscr{T}(C) \subseteq \mathscr{A}(C)+\mathscr{B}(C) .
$$

Since $\mathscr{T}$ is nondecreasing and partially continuous $\mathscr{T}(C)$ is again a bounded chain in $E$. Now, the partially Kurotowskii measure $\alpha^{p}$ of noncompactness is subadditive and full or complete and satisfies the properties $\left(5^{\circ}\right)$ and $\left(8^{\circ}\right)$ in $E$. As a result,

$$
\alpha^{p}(\mathscr{T} C) \leq \alpha^{p}(\mathscr{A}(C))+\alpha^{p}(\mathscr{B}(C)) \leq \psi\left(\alpha^{p}(C)\right)
$$

which shows that $\mathscr{T}$ is a partially nonlinear $\mathscr{D}$-set-contraction on $E$. Next, the order relation $\preceq$ and the norm $\|\cdot\|$ are compatible, so the desired conclusion follows by an application of Theorem 3.1. This completes the proof. 
Theorem 4.3. Let $(E, \preceq,\|\cdot\|)$ be a regular partially ordered complete normed linear space such that the order relation $\leq$ and the norm $\|\cdot\|$ in $E$ are compatible. Let $\mathscr{A}, \mathscr{B}: E \rightarrow E$ be two nondecreasing operators such that

(a) $\mathscr{A}$ is partially bounded and partially nonlinear $\mathscr{D}$-contraction,

(b) $\mathscr{B}$ is partially continuous and partially compact, and

(c) there exists an element $x_{0} \in E$ such that $x_{0} \geq \mathscr{A} x_{0}+\mathscr{B} x_{0}$.

Then the operator equation (4.1) has a solution $x^{*}$ in $E$ and the sequence $\left\{x_{n}\right\}$ of successive iterations defined by $x_{n+1}=\mathscr{A} x_{n}+\mathscr{B} x_{n}, n=0,1, \ldots ;$ converges monotonically to $x^{*}$.

\subsection{FPTs of Dhage type}

Let $(E, \preceq,\|\cdot\|)$ be a partially ordered normed linear algebra. Denote

$$
E^{+}=\{x \in E \mid x \geq \theta\} \quad \text { and } \quad \mathscr{K}=\left\{E^{+} \subset E \mid u \nu \in E^{+} \text {for all } u, v \in E^{+}\right\},
$$

where $\theta$ is the zero element of $E$. The members of $\mathscr{K}$ are called positive vectors in $E$.

Lemma 4.3 (Dhage [7]). If $u_{1}, u_{2}, v_{1}, v_{2} \in \mathscr{K}$ are such that $u_{1} \preceq v_{1}$ and $u_{2} \preceq v_{2}$, then $u_{1} u_{2} \preceq$ $v_{1} v_{2}$.

Definition 4.2. An operator $\mathscr{T}: E \rightarrow E$ is said to be positive if the range $R(\mathscr{T})$ of $\mathscr{T}$ is such that $R(\mathscr{T}) \subseteq \mathscr{K}$.

For any two chains $C_{1}$ and $C_{2}$ in $E$, denote

$$
C_{1} C_{2}=\left\{x \in E \mid x=c_{1} c_{2}, c_{1} \in C_{1} \text { and } c_{2} \in C_{2}\right\}
$$

Then we have the following lemma.

Lemma 4.4. If $C_{1}$ and $C_{2}$ are two bounded chains in a partially ordered normed linear algebra $E$, then

$$
\alpha^{p}\left(C_{1} C_{2}\right) \leq\left\|C_{2}\right\| \alpha^{p}\left(C_{1}\right)+\left\|C_{1}\right\| \alpha^{p}\left(C_{2}\right)
$$

where $\|C\|=\sup \{\|c\| \mid c \in C\}$ and $\alpha^{p}$ is a partially Kurotowskii measure of noncompactness in E.

Theorem 4.4. Let $(E, \preceq,\|\cdot\|)$ be a regular partially ordered complete normed linear algebra such that the order relation $\leq$ and the norm $\|\cdot\|$ in $E$ are compatible. Let $\mathscr{A}, \mathscr{B}: E \rightarrow \mathscr{K}$ and $\mathscr{C}: E \rightarrow E$ be three nondecreasing operators such that

(a) $\mathscr{A}$ and $\mathscr{C}$ are partially bounded and partially nonlinear $\mathscr{D}$-Lipschitz with $\mathscr{D}$-functions $\psi_{\mathscr{A}}$ and $\psi_{\mathscr{C}}$ respectively, 
(b) $\mathscr{B}$ is partially continuous and compact,

(c) $M \psi_{\mathscr{A}}(r)+\psi_{\mathscr{C}}(r)<r, r>0$, where $M=\|\mathscr{B}(E)\|$, and

(d) there exists an element $x_{0} \in X$ such that $x_{0} \leq \mathscr{A} x_{0} \mathscr{B} x_{0}+\mathscr{C} x_{0}$ or $x_{0} \geq \mathscr{A} x_{0} \mathscr{B} x_{0}+\mathscr{C} x_{0}$.

Then the operator equation

$$
\mathscr{A} x \mathscr{B} x+\mathscr{C} x=x
$$

has a solution $x^{*}$ in $E$ and the sequence $\left\{x_{n}\right\}$ of successive iterations defined by $x_{n+1}=\mathscr{A} x_{n} \mathscr{B} x_{n}+$ $\mathscr{C} x_{n}, n=0,1, \ldots ;$ converges monotonically to $x^{*}$.

Proof. Suppose that there exists an element $x_{0} \in X$ such that $x_{0} \leq \mathscr{A} x_{0} \mathscr{B} x_{0}+\mathscr{C} x_{0}$. Define a mapping $\mathscr{T}$ on $E$ by

$$
\mathscr{T} x=\mathscr{A} x \mathscr{B} x+\mathscr{C} x
$$

Since $\mathscr{A}$ and $\mathscr{B}$ are positive and $\mathscr{A}, \mathscr{B}$ and $\mathscr{C}$ are nondecreasing, $T$ is nondecreasing on $E$. From the partial continuity of the operators $\mathscr{A}, \mathscr{B}$ and $\mathscr{C}$ and the continuity of the binary compositions addition and multiplication, it follows that the operator $\mathscr{T}$ is partially continuous on $E$. As $\mathscr{A}$ and $\mathscr{C}$ are partially bounded and $\mathscr{B}$ is partially compact, we have that $\mathscr{T}$ is partially bounded on $E$. Again, by hypothesis (d), $x_{0} \leq \mathscr{T} x_{0}$. Next, we show that $\mathscr{T}$ is a partially nonlinear $\mathscr{D}$-set-contraction on $E$. Let $C$ be a bounded chain in $E$. Then by definition of $\mathscr{T}$, we have

$$
\mathscr{T}(C) \subseteq \mathscr{A}(C) \mathscr{B}(C)+\mathscr{C}(C)
$$

Since $\mathscr{T}$ is nondecreasing and partially continuous $\mathscr{T}(C)$ is again a bounded chain in $E$. Now, the partially Kurotowskii measure $\alpha^{p}$ of noncompactness in $E$ is subadditive and full or complete and satisfies the properties $\left(5^{\circ}\right)$ and $\left(8^{\circ}\right)$. As a result,

$$
\begin{aligned}
\alpha^{p}(\mathscr{T} C) & \leq\|\mathscr{A}(C)\| \alpha^{p}(\mathscr{B}(C))+\|\mathscr{B}(C)\| \alpha^{p}(\mathscr{A}(C))+\alpha^{p}(\mathscr{C}(C)) \\
& \leq\|\mathscr{B}(E)\| \alpha^{p}(\mathscr{A}(C))+\alpha^{p}(\mathscr{C}(C)) \\
& \leq M \psi_{\mathscr{A}}\left(\alpha^{p}(C)\right)+\psi_{\mathscr{C}}\left(\alpha^{p}(C)\right) \\
& =\psi\left(\alpha^{p}(C)\right),
\end{aligned}
$$

where $\psi(r)=M \psi_{\mathscr{A}}(r)+\psi_{\mathscr{C}}(r)<r, r>0$ and the constant $M$ exists in view of the fact that $\mathscr{B}$ is compact operator on $E$. This shows that $\mathscr{T}$ is a partially nonlinear $\mathscr{D}$-set-contraction on $E$. Next, the order relation $\preceq$ and the norm $\|\cdot\|$ are compatible, so the desired conclusion follows by an application of Theorem 3.1. Similarly, if $x_{0} \geq \mathscr{A} x_{0} \mathscr{B} x_{0}+\mathscr{C} x_{0}$, then using Theorem 3.2 it can be proved that $\mathscr{T}$ has a fixed point. This completes the proof.

Remark 4.1. If we take $\psi_{\mathscr{A}}(r)=\frac{L_{1} r}{K+r}$ and $\psi_{\mathscr{C}}(r)=L_{2} r$, then hypothesis (d) of the above hybrid fixed point theorem takes the form $\frac{L_{1} M}{K+r}+L_{2}<1$ for each real number $r>0$. Similarly, if $\psi_{\mathscr{A}}(r)=L_{1} r$, and $\psi_{\mathscr{C}}(r)=\frac{L_{2} r}{K+r}$, then hypothesis (d) of the above hybrid fixed point theorem takes the form $M L_{1}+\frac{L_{2} M}{K+r}<1$ for each real number $r>0$. 
Corollary 4.1. Let $(E, \preceq,\|\cdot\|)$ be a regular partially ordered complete normed linear algebra such that the order relation $\leq$ and the norm $\|\cdot\|$ in $E$ are compatible. Let $\mathscr{A}, \mathscr{B}: E \rightarrow \mathscr{K}$ be two nondecreasing operators such that

(a) $\mathscr{A}$ is partially bounded and partially nonlinear $\mathscr{D}$-Lipschitz with $\mathscr{D}$-function $\psi_{\mathscr{A}}$,

(b) $\mathscr{B}$ is partially continuous and compact,

(c) $M \psi_{\mathscr{A}}(r)<r, r>0$, where $M=\|\mathscr{B}(E)\|$ and

(d) there exists an element $x_{0} \in X$ such that $x_{0} \leq \mathscr{A} x_{0} \mathscr{B} x_{0}$ or $x_{0} \geq \mathscr{A} x_{0} \mathscr{B} x_{0}$.

Then the operator equation

$$
\mathscr{A} x \mathscr{B} x=x
$$

has a solution $x^{*}$ in $E$ and the sequence $\left\{x_{n}\right\}$ of successive iterations defined by $x_{n+1}=\mathscr{A} x_{n} \mathscr{B} x_{n}$, $n=0,1, \ldots ;$ converges monotonically to $x^{*}$.

Remark 4.2. The hypotheses (b) and (c) in Theorem 4.4 may be replaced with the weaker hypotheses as follows:

$\left(\mathrm{b}^{\prime}\right) \mathscr{B}$ is partially continuous and uniformly partially compact, and

(c') $M \psi_{\mathscr{A}}(r)+\psi_{\mathscr{C}}(r)<r, r>0$, where $M=\sup \left\{\|\mathscr{B}(C)\|: C \in \mathscr{P}_{c h}\right\}$.

The proof of Theorem 4.4 under these new hypotheses is essentially the same as that given in the theorem. Similarly, the conclusion of Corollary 4.1 also remains true under the corresponding changes in the hypotheses (b) and (c) thereof. Corollary 4.1 is useful in the study of quadratic nonlinear differential and integral equations for qualitative aspects of the solutions.

Remark 4.3. The conclusion of hybrid fixed point theorems of Sections 3 and 4 also remains true if we replace the compatibility of $E$ with respect to the order relation $\leq$ and the norm $\|\cdot\|$ by the weaker condition of the compatibility of every compact chain $C$ in $E$. The later condition holds if $\leq$ and $\|\cdot\|$ are compatible in every partially compact subset of $E$.

\section{Functional integral equations}

In this section, we are going to prove a result on the existence and uniform global attractivity of comparable solutions for a nonlinear functional integral equation. Our investigations will be carried out in the partially ordered Banach space of real functions which are defined, continuous and bounded on the right half real axis $\mathbb{R}_{+}$. The integral equation in question has rather general form and contains as particular cases a lot of functional equations and nonlinear integral equations of Volterra type. The main tool used in our considerations is the technique of partially measures of noncompactness and the fixed point result established in Theorem 3.1. The measure of noncompactness used in this paper allows us not only to obtain 
the existence of solutions of the mentioned functional integral equation but also to characterize the comparable solutions in terms of uniform global ultimate attractivity. This assertion means that all possible comparable solutions of the functional integral equation in question are globally uniformly ultimately attractive in the sense of notion defined in the following section.

\subsection{Notation, definitions and auxiliary facts}

Our considerations will be placed in the Banach space $B C\left(\mathbb{R}_{+}, \mathbb{R}\right)$ consisting of all real functions $x=x(t)$ defined, continuous and bounded on $\mathbb{R}_{+}$. This space is equipped with the standard supremum norm

$$
\|x\|=\sup \left\{|x(t)|: t \in \mathbb{R}_{+}\right\} .
$$

Define the order relation $\leq$ in $B C\left(\mathbb{R}_{+}, \mathbb{R}\right)$ as follows. Let $x, y \in B C\left(\mathbb{R}_{+}, \mathbb{R}\right)$. Then by $x \leq$ $y$ we mean $x(t) \leq y(t)$ forall $t \in \mathbb{R}_{+}$. It is clear that $\left(B C\left(\mathbb{R}_{+}, \mathbb{R}\right), \leq,\|\cdot\|\right)$ is a regular partially ordred Banach space and the order relation $\leq$ and the norm $\|\cdot\|$ are compatible in every partially compact subset of $B C\left(\mathbb{R}_{+}, \mathbb{R}\right)$. Further $\left(B C\left(\mathbb{R}_{+}, \mathbb{R}\right), \leq\right)$ is also a lattice so that every pair of elements in it has a least lower bound and a greatest upper bound. (cf. Nieto and Lopez [19]). See also Carl and Heikkilä [5] and the references therein.

For our purposes we introduce a measure of noncompactness which is a handy tool in the study of the solutions of certain nonlinear integral equations. To define this partial measure, let us fix a nonempty and bounded chain $X$ of the space $B C\left(\mathbb{R}_{+}, \mathbb{R}\right)$ and a positive number $T$. For $x \in X$ and $\epsilon \geq 0$ denote by $\omega^{T}(x, \epsilon)$ the modulus of continuity of the function $x$ on the interval $[0, T]$ defined by

$$
\omega^{T}(x, \epsilon)=\sup \{|x(t)-x(s)|: t, s \in[0, T],|t-s| \leq \epsilon\} .
$$

Next, let us put

$$
\begin{aligned}
\omega^{T}(X, \epsilon) & =\sup \left\{\omega^{T}(x, \epsilon): x \in X\right\}, \\
\omega_{0}^{T}(X) & =\lim _{\epsilon \rightarrow 0} \omega^{T}(X, \epsilon), \\
\omega_{0}(X) & =\lim _{T \rightarrow \infty} \omega_{0}^{T}(X) .
\end{aligned}
$$

It is claimed that the partial Hausdorff measure of noncompactness $\beta^{p}$ is very much useful in applications to nonlinear differential and integral equations and it can be shown that

$$
\beta^{p}(X)=\frac{1}{2} \omega_{0}(X)
$$

for all bounded chain $X$ in $E$. Thus $\omega_{0}$ is a handy tool for $\beta^{p}$ in $E$. 
Now, for a fixed number $t \in \mathbb{R}_{+}$and a fixed bounded chain $X$ in $B C\left(\mathbb{R}_{+}, \mathbb{R}\right)$, let us denote

$$
X(t)=\{x(t): x \in X\}
$$

Define

and

$$
\begin{aligned}
& \delta_{a}(X(t))=|X(t)|=\sup \{|x(t)|: x \in X\} \\
& \delta_{a}^{T}(X(t))=\sup _{t \geq T} \delta_{a}(X(t))=\sup _{t \geq T}|X(t)|
\end{aligned}
$$

$$
\delta_{a}(X)=\lim _{T \rightarrow \infty} \delta_{a}^{T}(X(t))=\limsup _{t \rightarrow \infty}|X(t)|
$$

Again, for a fixed real number $c$, denote

$$
X(t)-c=\{x(t)-c: x \in X\}
$$

Define

and

$$
\begin{aligned}
& \delta_{b}(X(t))=|X(t)-c|=\sup \{|x(t)-c|: x \in X\} \\
& \delta_{b}^{T}(X(t))=\sup _{t \geq T} \delta_{b}(X(t))=\sup _{t \geq T}|X(t)-c|
\end{aligned}
$$

$$
\delta_{b}(X)=\lim _{T \rightarrow \infty} \delta_{b}^{T}(X(t))=\limsup _{t \rightarrow \infty}|X(t)-c|
$$

Similarly, let

and

$$
\begin{aligned}
& \delta_{c}(X(t))=\operatorname{diam} X(t)=\sup \{|x(t)-y(t)|: x, y \in X\} \\
& \delta_{c}^{T}(X(t))=\sup _{t \geq T} \delta(X(t))=\sup _{t \geq T} \operatorname{diam} X(t)
\end{aligned}
$$

$$
\delta_{c}(X)=\lim _{T \rightarrow \infty} \delta^{T}(X(t))=\limsup _{t \rightarrow \infty} \operatorname{diam} X(t) .
$$

The details of the functions $\delta_{a}, \delta_{b}$ and $\delta_{c}$ appear in Dhage [8]. Finally, let us consider the functions $\mu_{a}^{p}, \mu_{b}^{p}$ and $\mu_{c}^{p}$ defined on the family of bounded chains in $B C\left(\mathbb{R}_{+}, \mathbb{R}\right)$ by the formula

$$
\begin{aligned}
& \mu_{a}^{p}(X)=\max \left\{\omega_{0}(X), \delta_{a}(X)\right\} \\
& \mu_{b}^{p}(X)=\max \left\{\omega_{0}(X), \delta_{b}(X)\right\}
\end{aligned}
$$

and

$$
\mu_{c}^{p}(X)=\max \left\{\omega_{0}(X), \delta_{c}(X)\right\}
$$

It can be shown that the functions $\mu_{a}^{p}, \mu_{b}^{p}$ and $\mu_{c}^{p}$ are partially measures of noncompactness in the space $B C\left(\mathbb{R}_{+}, \mathbb{R}\right)$. The components $\omega_{0}$ and $\delta_{a}$ are called the characteristic values of the partially measure of noncompactness $\mu_{a}^{p}$. Similarly, $\omega_{0}, \delta_{b}$ and $\omega_{0}, \delta_{c}$ are respectively the characteristic values of the partially measure of noncompactness $\mu_{b}^{p}$ and $\mu_{c}^{p}$ in $B C\left(\mathbb{R}_{+}, \mathbb{R}\right)$. 
Remark 5.1. The kernels $\operatorname{ker} \mu_{a}^{p}$, $\operatorname{ker} \mu_{b}^{p}$ and $\operatorname{ker} \mu_{c}^{p}$ consist of nonempty and bounded chains $X$ of $B C\left(\mathbb{R}_{+}, \mathbb{R}\right)$ such that functions from $X$ are locally equicontinuous on $\mathbb{R}_{+}$and the thickness of the bundle formed by functions from $X$ tends to zero at infinity. This particular characteristic of $\operatorname{ker} \mu_{a}^{p}$, $\operatorname{ker} \mu_{b}^{p}$ and $\operatorname{ker} \mu_{c}^{p}$ has been useful in establishing the global attractivity and global asymptotic stability of the comparable solutions for nonlinear functional integral equations.

In order to introduce further concepts used in the paper let us assume that $\Omega$ is a nonempty chain of the space $B C\left(\mathbb{R}_{+}, \mathbb{R}\right)$. Moreover, let $Q$ be an operator defined on $\Omega$ with values in $B C\left(\mathbb{R}_{+}, \mathbb{R}\right)$.

Consider the operator equation of the form

$$
x(t)=Q x(t), t \in \mathbb{R}_{+} .
$$

Definition 5.1. We say that comparable solutions of the equation (5.5) are locally attractive if there exists a ball $\overline{\mathscr{B}}\left(x_{0}, r\right)$ in the space $B C\left(\mathbb{R}_{+}, \mathbb{R}\right)$ such that for arbitrary comparable solutions $x=x(t)$ and $y=y(t)$ of the equation (5.5) belonging to $\overline{\mathscr{B}}\left(x_{0}, r\right) \cap \Omega$ we have that

$$
\lim _{t \rightarrow \infty}[x(t)-y(t)]=0 .
$$

In the case when limit (5.6) is uniform with respect to the set $\overline{\mathscr{B}}\left(x_{0}, r\right) \cap \Omega$, i.e. when for each $\epsilon>0$ there exists $T>0$ such that

$$
|x(t)-y(t)| \leq \epsilon
$$

for all $x, y \in \overline{\mathscr{B}}\left(x_{0}, r\right) \cap \Omega$ being the comparable solutions of (5.5) and for $t \geq T$, we will say that the comparable solutions of the operator equation (5.5) are uniformly locally ultimately attractive defined on $\mathbb{R}_{+}$.

Definition 5.2. A comparable solution $x=x(t)$ of equation (5.5) is said to be globally ultimately attractive if (5.6) holds for every comparable solution $y=y(t)$ of (5.5) on $\mathbb{R}_{+}$. In other words, we may say that the comparable solutions of the equation (5.5) are globally ultimately attractive if for arbitrary comparable solutions $x(t)$ and $y(t)$ of (5.5) the condition (5.6) is satisfied. In the case when condition (5.6) is satisfied uniformly with respect to the set $B C\left(\mathbb{R}_{+}, \mathbb{R}\right)$, i.e., if for every $\epsilon>0$ there exists $T>0$ such that the inequality (5.7) is satisfied for all $x, y \in \Omega$ being the comparable solutions of (5.5) and for $t \geq T$, we will say that the comparable solutions of the equation (5.5) are uniformly globally ultimately attractive on $\mathbb{R}_{+}$.

Let us mention that the concept of asymptotic stability may be found in Banas and Dhage [3] and references therein wheres the concept of global attractivity of solutions is introduced 
in Dhage [8] and proved attractivity results for certain nonlinear integral equations. We mention that the present approach is constructive and different from that given in the above stated papers.

\subsection{Integral equation and attractivity result}

Now we will investigate the following nonlinear functional integral equation (in short FIE)

$$
x(t)=f(t, x(\alpha(t)))+\int_{0}^{\beta(t)} g(t, s, x(\gamma(s))) d s
$$

for all $t \in \mathbb{R}_{+}$, where the functions $f: \mathbb{R}_{+} \times \mathbb{R} \rightarrow \mathbb{R}$ and $g: \mathbb{R}_{+} \times \mathbb{R}_{+} \times \mathbb{R} \rightarrow \mathbb{R}$ are continuous and $\alpha, \beta, \gamma: \mathbb{R}_{+} \rightarrow \mathbb{R}_{+}$.

By a solution of the FIE (5.8) we mean a function $x \in C\left(\mathbb{R}_{+}, \mathbb{R}\right)$ that satisfies the equation (5.8), where $C\left(\mathbb{R}_{+}, \mathbb{R}\right)$ is the space of continuous real-valued functions on $\mathbb{R}_{+}$.

Observe that the above integral equation has been discussed in Banas and Dhage [3] under strong Lipschitz condition for the attractivity of solutions and includes several classes of functional, integral and functional integral equations considered in the literature (cf. $[1,4,7]$ and references therein). Let us also mention that the functional integral equation considered in $[3,4]$ is a special case of the equation (5.8), where $\alpha(t)=\beta(t)=\gamma(t)=t$. The novelty of the present approach lies in the fact that we use a weaker one sided or partially Lipschitz condition and derive some stronger conclusions for comparable solutions.

The nonlinear integral equation (5.8) will be considered under the following assumptions:

$\left(\mathrm{H}_{1}\right)$ The functions $\alpha, \beta, \gamma: \mathbb{R}_{+} \rightarrow \mathbb{R}_{+}$are continuous and $\alpha(t) \geq t$ for all $t \in \mathbb{R}_{+}$.

$\left(\mathrm{H}_{2}\right)$ The function $F: \mathbb{R}_{+} \rightarrow \mathbb{R}_{+}$defined by $F(t)=|f(t, 0)|$ is bounded on $\mathbb{R}_{+}$with $F_{0}=\sup _{t \geq 0} F(t)$.

$\left(\mathrm{H}_{3}\right)$ There exist constants $L>0$ and $K>0$ such that

$$
0 \leq f(t, x)-f(t, y) \leq \frac{L(x-y)}{K+(x-y)}
$$

for all $t \in \mathbb{R}_{+}$and $x, y \in \mathbb{R}$ with $x \geq y$. Moreover, $L \leq K$.

$\left(\mathrm{H}_{4}\right) g(t, s, x)$ is nondecreasing in $x$ for each $t, s \in \mathbb{R}_{+}$.

$\left(\mathrm{H}_{5}\right)$ There exists an element $u \in B C\left(\mathbb{R}_{+}, \mathbb{R}\right)$ such that

$$
\left.u(t) \leq f(t, u(\alpha(t)))+\int_{t_{0}}^{\beta(t))} g(t, s, u(\gamma(s)))\right) d s
$$

for all $t \in \mathbb{R}_{+}$. 
$\left(\mathrm{H}_{6}\right)$ The function $g: \mathbb{R}_{+} \times \mathbb{R}_{+} \times \mathbb{R} \rightarrow \mathbb{R}$ is continuous and there exist functions $a, b: \mathbb{R}_{+} \rightarrow \mathbb{R}_{+}$ such that

$$
|g(t, s, x)| \leq a(t) b(s)
$$

for $t, s \in \mathbb{R}_{+}$and $x \in \mathbb{R}$. Moreover, we assume that

$$
\lim _{t \rightarrow \infty} a(t) \int_{0}^{\beta(t)} b(s) d s=0 .
$$

$\left(\mathrm{H}_{7}\right)$ There exists a real number $c$ such that $f(t, c)=c$ for all $t \in \mathbb{R}_{+}$.

The hypotheses $\left(\mathrm{H}_{1}\right)-\left(\mathrm{H}_{2}\right)$ and $\left(\mathrm{H}_{4}\right),\left(\mathrm{H}_{6}\right)$ have been widely used in the literature on nonlinear differential and integral equations. The special case of hypothesis $\left(\mathrm{H}_{3}\right)$ with $L<K$ is considered in Nieto and Lopez [19]. Now we formulate the main existence result for the integral equation (5.6) under above mentioned natural conditions.

Theorem 5.1. Assume that the hypotheses $\left(\mathrm{H}_{1}\right)$ through $\left(\mathrm{H}_{6}\right)$ hold. Then the functional integral equation (5.8) has at least one solution $x^{*}$ in the space $B C\left(\mathbb{R}_{+}, \mathbb{R}\right)$ and the sequence $\left\{x_{n}\right\}$ of successive approximations defined by

$$
x_{n}(t)=f\left(t, x_{n-1}(\alpha(t))\right)+\int_{0}^{\beta(t)} g\left(t, s, x_{n-1}(\gamma(s))\right) d s
$$

for all $t \in \mathbb{R}_{+}$with $x_{0}=u$ converges monotonically to $x^{*}$. Moreover, the comparable solutions of the equation (5.8) are uniformly globally ultimately attractive on $\mathbb{R}_{+}$.

Proof. We seek the solutions of the FIE (5.8) in the space $B C\left(\mathbb{R}_{+}, \mathbb{R}\right)$ of continuous and bounded real-valued functions defined on $\mathbb{R}_{+}$. Set $E=B C\left(\mathbb{R}_{+}, \mathbb{R}\right)$. Define an order relation $\leq$ in $E$ as $x \leq y$ if and only if $x(t) \leq y(t)$ for all $t \in \mathbb{R}_{+}$. Clearly, $E$ is regular and the order relation $\leq$ an the norm $\|\cdot\|$ in $E$ are compatible in every compact chain $C$ of $E$. Consider the operator $Q$ defined on the space $B C\left(\mathbb{R}_{+}, \mathbb{R}\right)$ by the formula

$$
Q x(t)=f(t, x(\alpha(t)))+\int_{0}^{\beta(t)} g(t, s, x(\gamma(s))) d s, t \in \mathbb{R}_{+} .
$$

Observe that in view of our assumptions, for any function $x \in E$ the function $Q x$ is continuous on $\mathbb{R}_{+}$. As a result, $Q$ defines a mapping $Q: E \rightarrow E$. We show that $Q$ satisfies all the conditions of Theorem 3.1 on $E$. This will be achieved in a series of following steps:

Step I: $Q$ is nondecreasing on $E$.

Let $x, y \in E$ be such that $x \leq y$. Then by hypothesis $\left(\mathrm{H}_{3}\right)-\left(\mathrm{H}_{4}\right)$, we obtain

$$
Q x(t)=f(t, x(\alpha(t)))+\int_{0}^{\beta(t)} g(t, s, x(\gamma(s))) d s
$$




$$
\begin{aligned}
& \leq f(t, y(\alpha(t)))+\int_{0}^{\beta(t)} g(t, s, y(\gamma(s))) d s \\
& =Q y(t)
\end{aligned}
$$

for all $t \in \mathbb{R}_{+}$. This shows that $Q$ is a nondecreasing operator on $E$.

Step II: $Q$ is partially bounded on $E$.

Let $X$ be a chain in $E$ and let $x \in X$. Since the function $v: \mathbb{R}_{+} \rightarrow \mathbb{R}$ defined by

$$
v(t)=\lim _{t \rightarrow \infty} a(t) \int_{0}^{\beta(t)} b(s) d s
$$

is continuous and in view of hypothesis $\left(\mathrm{H}_{6}\right)$, the number $V=\sup _{t \geq 0} v(t)$ exists. Moreover if $x \geq \theta$, then for arbitrarily fixed $t \in \mathbb{R}_{+}$we obtain:

$$
\begin{aligned}
|Q x(t)| & \leq|f(t, x(\alpha(t)))|+\int_{0}^{\beta(t)}|g(t, s, x(s))| d s \\
& \leq|f(t, x(\alpha(t)))-f(t, 0)|+|f(t, 0)|+a(t) \int_{0}^{\beta(t)} b(s) d s \\
& \leq \frac{L|x(\alpha(t))|}{K+|x(\alpha(t))|}+F(t)+v(t) \\
& \leq \frac{L\|x\|}{K+\|x\|}+F_{0}+V=L+F_{0}+V=r .
\end{aligned}
$$

Similarly, if $x \geq \theta$, then it can be shown that $|Q x(t)| \leq r$ for all $t \in \mathbb{R}_{+}$. Taking the suremum over $t$, we obtain $\|Q x\| \leq r$ for all $x \in X$. This means that the operator $Q$ transforms any chain $X$ into a bounded chain in $E$. More precisely, we obtain that the operator $Q$ transforms the chain $X$ into the chain $Q(X)$ contained in the ball $\overline{\mathscr{B}}(\theta, r)$, where $r=L+F_{0}+V$. As a result, $Q$ defines a mapping $Q: \mathscr{P}_{c h}(E) \rightarrow \mathscr{P}_{c h}(\overline{\mathscr{B}}(\theta, r))$ and that $Q$ is partially bounded on $E$.

Step III: $Q$ is partially continuous on E.

Now we show that the operator $Q$ is partially continuous on $E$. To do this, let $X$ be a chain in $E$ and let us fix arbitrarily $\epsilon>0$ and take $x, y \in X$ such that $x \geq y$ and $\|x-y\| \leq \epsilon$. Then we get:

$$
\begin{aligned}
|Q x(t)-Q y(t)| \leq & |f(t, x(\alpha(t)))-f(t, y(\alpha(t)))| \\
& +\left|\int_{0}^{\beta(t)} g(t, s, x(\gamma(s))) d s-\int_{0}^{\beta(t)} g(t, s, y(\gamma(s))) d s\right| \\
\leq & |f(t, x(\alpha(t)))-f(t, y(\alpha(t)))| \\
& +\int_{0}^{\beta(t)}|g(t, s, x(\gamma(s)))| d s+\int_{0}^{\beta(t)}|g(t, s, y(\gamma(s)))| d s \\
\leq & \frac{L|x(\alpha(t))-y(\alpha(t))|}{K+|x(\alpha(t))-y(\alpha(t))|}+2 a(t) \int_{0}^{\beta(t)} b(s) d s
\end{aligned}
$$




$$
\begin{aligned}
& \leq \frac{L\|x-y\|}{K+\|x-y\|}+2 v(t) \\
& <\epsilon+2 v(t) .
\end{aligned}
$$

Hence, in virtue of hypothesis $\left(\mathrm{H}_{6}\right)$ we infer that there exists $T>0$ such tha $\nu(t) \leq \frac{\epsilon}{2}$ for $t \geq T$. Thus, for $t \geq T$ we derive that

$$
|Q x(t)-Q y(t)|<2 \epsilon .
$$

Further, let us assume that $t \in[0, T]$. Then, evaluating similarly as above we get:

$$
\begin{aligned}
|Q x(t)-Q y(t)| \leq & |f(t, x(\alpha(t)))-f(t, y(\alpha(t)))| \mid \\
& +\int_{0}^{\beta(t)}[|g(t, s, x(\gamma(s)))-g(t, s, y(\gamma(s)))|] d s \\
\leq & \frac{L|x(\alpha(t))-y(\alpha(t))|}{K+|x(\alpha(t))-y(\alpha(t))|} \\
& +\int_{0}^{\beta_{T}}[|g(t, s, x(\gamma(s)))-g(t, s, y(\gamma(s)))|] d s \\
< & \epsilon+\beta_{T} \omega_{r}^{T}(g, \epsilon),
\end{aligned}
$$

where we have denoted

$$
\beta_{T}=\sup \{\beta(t): t \in[0, T]\}
$$

and

$$
\omega_{r}^{T}(g, \epsilon)=\sup \{|g(t, s, x)-g(t, s, y)|: t, s \in[0, T], x, y \in[-r, r],|x-y| \leq \epsilon\} .
$$

Obviously, in view of continuity of $\beta$, we have that $\beta_{T}<\infty$. Moreover, from the uniform continuity of the function $g(t, s, x)$ on the set $[0, T] \times[0, T] \times[-r, r]$ we derive that $\omega_{r}^{T}(g, \epsilon) \rightarrow 0$ as $\epsilon \rightarrow 0$. Now, linking (5.13), (5.14) and the above established facts we conclude that the operator $Q$ maps partially continuously the ball $\overline{\mathscr{B}}(\theta, r)$ into itself.

Step IV: $Q$ is a nonlinear $\mathscr{D}$-set-contraction w.r.t. the characteristic value $\omega_{0}$.

Further on let us take a bounded chain $X$ in $E$ with bound $r>0$, i.e., the chain $X$ belonging to the ball $\mathscr{B}(\theta, r)$. Next, fix arbitrarily $T>0$ and $\epsilon>0$. Let us choose $x \in X$ and $t_{1}, t_{2} \in[0, T]$ with $\left|t_{2}-t_{1}\right| \leq \epsilon$. Without loss of generality we may assume that $x\left(\alpha\left(t_{1}\right)\right) \geq x\left(\alpha\left(t_{2}\right)\right)$. Then, taking into account our assumptions, we get:

$$
\begin{aligned}
\left|Q x\left(t_{1}\right)-Q x\left(t_{2}\right)\right| \leq & \left|f\left(t_{1}, x\left(\alpha\left(t_{1}\right)\right)\right)-f\left(t_{2}, x\left(\alpha\left(t_{2}\right)\right)\right)\right| \\
& +\left|\int_{0}^{\beta\left(t_{1}\right)} g\left(t_{1}, s, x(\gamma(s))\right) d s-\int_{0}^{\beta\left(t_{2}\right)} g\left(t_{2}, s, x(\gamma(s))\right) d s\right| \\
\leq & \left|f\left(t_{1}, x\left(\alpha\left(t_{1}\right)\right)\right)-f\left(t_{2}, x\left(\alpha\left(t_{2}\right)\right)\right)\right|
\end{aligned}
$$




$$
\begin{aligned}
& \quad+\left|\int_{0}^{\beta\left(t_{1}\right)} g\left(t_{1}, s, x(\gamma(s))\right) d s-\int_{0}^{\beta\left(t_{2}\right)} g\left(t_{2}, s, x(\gamma(s))\right) d s\right| \\
& +\left|\int_{0}^{\beta\left(t_{1}\right)} g\left(t_{1}, s, x(\gamma(s))\right) d s-\int_{0}^{\beta\left(t_{2}\right)} g\left(t_{2}, s, x(\gamma(s))\right) d s\right| \\
& \leq\left|f\left(t_{1}, x\left(\alpha\left(t_{1}\right)\right)\right)-f\left(t_{2}, x\left(\alpha\left(t_{2}\right)\right)\right)\right| \\
& +\int_{0}^{\beta\left(t_{1}\right)}\left|g\left(t_{1}, s, x(\gamma(s))\right)-g\left(t_{2}, s, x(\gamma(s))\right)\right| d s \\
& +\left|\int_{\beta\left(t_{2}\right)}^{\beta\left(t_{1}\right)}\right| g\left(t_{2}, s, x(\gamma(s))\right)|d s| \\
& \leq\left|f\left(t_{1}, x\left(\alpha\left(t_{1}\right)\right)\right)-f\left(t_{2}, x\left(\alpha\left(t_{2}\right)\right)\right)\right| \\
& +\int_{0}^{\beta}\left|g\left(t_{1}, s, x(\gamma(s))\right)-g\left(t_{2}, s, x(\gamma(s))\right)\right| d s+G_{T}^{r}\left|\beta\left(t_{1}\right)-\beta\left(t_{2}\right)\right|,
\end{aligned}
$$

where

$$
G_{T}^{r}=\sup \left\{|g(t, s, x)|: t \in[0, T], s \in\left[0, \beta_{T}\right], x \in[-r, r]\right\}
$$

which does exist in view of continuity of the function $g$ on compact $[0, T] \times\left[0, \beta_{T}\right] \times[-r, r]$.

Now combining (5.14) and (5.15) we obtain,

$$
\begin{aligned}
\left|Q x\left(t_{2}\right)-Q x\left(t_{1}\right)\right| \leq & \left|f\left(t_{1}, x\left(\alpha\left(t_{1}\right)\right)\right)-f\left(t_{2}, x\left(\alpha\left(t_{1}\right)\right)\right)\right|+\frac{L\left|x\left(\alpha\left(t_{1}\right)\right)-x\left(\alpha\left(t_{2}\right)\right)\right|}{K+\left|x\left(\alpha\left(t_{1}\right)\right)-x\left(\alpha\left(t_{2}\right)\right)\right|} \\
& +\int_{0}^{\beta_{T}}\left|g\left(t_{1}, s, x(\gamma(s))\right)-g\left(t_{2}, s, x(\gamma(s))\right)\right| d s+G_{T}^{r}\left|\beta\left(t_{1}\right)-\beta\left(t_{2}\right)\right| \\
\leq & \frac{L \omega^{T}\left(x, \omega^{T}(\alpha, \epsilon)\right)}{K+\omega^{T}\left(x, \omega^{T}(\alpha, \epsilon)\right)}+\omega_{r}^{T}(f, \epsilon)+\int_{0}^{\beta_{T}} \omega_{r}^{T}(g, \epsilon) d s+G_{T}^{r} \omega^{T}(\beta, \epsilon),
\end{aligned}
$$

where we have denoted

$$
\begin{aligned}
& \omega^{T}(\alpha, \epsilon)=\sup \left\{\left|\alpha\left(t_{2}\right)-\alpha\left(t_{1}\right)\right|: t_{1}, t_{2} \in[0, T],\left|t_{2}-t_{1}\right| \leq \epsilon\right\}, \\
& \omega^{T}(\beta, \epsilon)=\sup \left\{\left|\beta\left(t_{2}\right)-\beta\left(t_{1}\right)\right|: t_{1}, t_{2} \in[0, T],\left|t_{2}-t_{1}\right| \leq \epsilon\right\}, \\
& \omega_{r}^{T}(f, \epsilon)=\sup \left\{\left|f\left(t_{2}, x\right)-f\left(t_{1}, x\right)\right|: t_{1}, t_{2} \in[0, T],\left|t_{2}-t_{1}\right| \leq \epsilon, x \in[-r, r]\right\},
\end{aligned}
$$

and

$$
\omega_{r}^{T}(g, \epsilon)=\sup \left\{\left|g\left(t_{2}, s, x\right)-g\left(t_{1}, s, x\right)\right|: t_{1}, t_{2} \in[0, T],\left|t_{2}-t_{1}\right| \leq \epsilon, \quad s \in\left[0, \beta_{T}\right], x \in[-r, r]\right\} .
$$

From the above estimate we derive the following one:

$$
\omega^{T}(Q(X), \epsilon) \leq \frac{L \omega^{T}\left(X, \omega^{T}(\alpha, \epsilon)\right)}{K+\omega^{T}\left(X, \omega^{T}(\alpha, \epsilon)\right)}+\omega_{r}^{T}(f, \epsilon)+\int_{0}^{\beta_{T}} \omega_{r}^{T}(g, \epsilon) d s+G_{T}^{r} \omega^{T}(\beta, \epsilon) .
$$

Observe that $\omega_{r}^{T}(f, \epsilon) \rightarrow 0$ and $\omega_{r}^{T}(g, \epsilon) \rightarrow 0$ as $\epsilon \rightarrow 0$, which is a simple consequence of the uniform continuity of the functions $f$ and $g$ on the sets $[0, T] \times[-r, r]$ and $[0, T] \times\left[0, \beta_{T}\right] \times[-r, r]$ 
respectively. Moreover, from the uniform continuity of $\alpha, \beta$ on $[0, T]$, it follows that $\omega^{T}(\alpha, \epsilon) \rightarrow$ $0, \omega^{T}(\beta, \epsilon) \rightarrow 0$ as $\epsilon \rightarrow 0$. Thus, linking the established facts with the estimate (5.14) we get

$$
\omega_{0}^{T}(Q(X)) \leq \frac{L \omega_{0}^{T}(X)}{K+\omega_{0}^{T}(X)} .
$$

Consequently, we obtain

$$
\omega_{0}(Q(X)) \leq \frac{L \omega_{0}(X)}{K+\omega_{0}(X)}
$$

Step V: $Q$ is a nonlinear $\mathscr{D}$-set-contraction w.r.t. characteristic value $\delta_{c}$.

Now, taking into account our assumptions, for arbitrarily fixed $t \in \mathbb{R}_{+}$and for $x, y \in X$ with $x \geq y$, we deduce the following estimate:

$$
\begin{aligned}
|(Q x)(t)-(Q y)(t)| & \leq|f(t, x(\alpha(t)))-f(t, y(\alpha(t)))|+2\left(a(t) \int_{0}^{\beta(t)} b(s) d s\right) \\
& \leq \frac{L|x(\alpha(t))-y(\alpha(t))|}{K+|x(\alpha(t))-y(\alpha(t))|}+2 v(t) .
\end{aligned}
$$

From the above inequality it follows that

$$
\operatorname{diam}(Q X(t)) \leq \frac{L \operatorname{diam}(X(\alpha(t)))}{K+\operatorname{diam}(X(\alpha(t)))}+v(t)
$$

for each $t \in \mathbb{R}_{+}$. Therefore, taking limit superior over $t \rightarrow \infty$, we obtain

$$
\begin{aligned}
\delta_{c}(Q X) & =\limsup _{t \rightarrow \infty} \operatorname{diam}(Q(X(t))) \\
& \leq \frac{L \limsup \sup _{t \rightarrow \infty} \operatorname{diam}(X(\alpha(t)))}{K+\limsup \operatorname{sum}_{t \rightarrow \infty} \operatorname{diam} X(\alpha(t))} \\
& \leq \frac{L \limsup \sup _{t \rightarrow \infty} \operatorname{diam}(X(t))}{K+\limsup } \operatorname{diam}_{t \rightarrow \infty}(X(t)) \\
& =\frac{L \delta_{c}(X)}{K+\delta_{c}(X)} .
\end{aligned}
$$

Step VI: $Q$ is a partially nonlinear $\mathscr{D}$-set-contraction on E.

Further, using the measure of noncompactness $\mu_{c}^{p}$ defined by the formula (5.4) and keep- 
ing in mind the estimates (5.18) and (5.19), we obtain

$$
\begin{aligned}
\mu_{c}^{p}(Q X) & =\max \left\{\omega_{0}(Q X), \delta_{c}(Q X)\right\} \\
& \leq \max \left\{\frac{L \omega_{0}(X)}{K+\omega_{0}(X)}, \frac{L \delta_{c}(X)}{K+\delta_{c}(X)}\right\} \\
& \leq \frac{L \max \left\{\omega_{0}(X), \delta_{c}(X)\right\}}{K+\max \left\{\omega_{0}(X), \delta_{c}(X)\right\}} \\
& =\frac{L \mu_{c}^{p}(X)}{K+\mu_{c}^{p}(X)}
\end{aligned}
$$

for all bounded chains $X$ in $E$. Since $L \leq K$, the operator $Q$ is a partially nonlinear $\mathscr{D}$-setcontraction on $E$ with $\mathscr{D}$-function $\psi(r)=\frac{L r}{K+r}$. Again, by hypothesis $\left(\mathrm{H}_{5}\right)$, there exists an element $x_{0}=u \in E$ such that $x_{0} \leq Q x_{0}$, that is, $x_{0}$ is a lower solution of the FIE (5.8) defined on $\mathbb{R}_{+}$.

Thus $Q$ satisfies all the conditions of Theorem 3.1 on $E$ in view of Remark 4.3. Hence we apply it to the operator equation $Q x=x$ and deduce that the operator $Q$ has a fixed point $x^{*}$ in $E$. Obviously $x^{*}$ is a solution of the functional integral equation (5.8) and the sequence $\left\{x_{n}\right\}$ of successive approximations defined by (5.9) converges monotonically to $x^{*}$. Moreover, taking into account that the image of every chain $X$ under the operator $Q$ is again a chain $Q(X)$ contained in the ball $\overline{\mathscr{B}}(\theta, r)$ we infer that the set $\mathscr{F}(Q)$ of all fixed points of $Q$ is contained in $\overline{\mathscr{B}}(\theta, r)$. If the set $\mathscr{F}(Q)$ contains all comparable solutions of the equation (5.8), then we conclude from Remark 3.3 that the set $\mathscr{F}(Q)$ belongs to the family $\operatorname{ker} \mu_{c}^{p}$. Now, taking into account the description of sets belonging to ker $\mu_{c}^{p}$ (given in Section 3) we deduce that all comparable solutions of the equation (5.8) are uniformly globally ultimately attractive on $\mathbb{R}_{+}$. This completes the proof.

Theorem 5.2. Assume that the hypotheses $\left(H_{1}\right)$ through $\left(H_{7}\right)$ hold. Then the functional FIE (5.8) has at least one solution $x^{*}$ in the space $B C\left(\mathbb{R}_{+}, \mathbb{R}\right)$ and the sequence $\left\{x_{n}\right\}$ of successive approximations defined by (5.9) converges monotonically to $x^{*}$. Moreover, the comparable solutions of the equation (5.8) are uniformly globally ultimately asymptotically stable to the line $x(t)=c$ defined on $\mathbb{R}_{+}$.

Proof. As in Theorem 5.1, we seek the solutions of the FIE (5.8) in the space $E=B C\left(\mathbb{R}_{+}, \mathbb{R}\right)$. Define the closed bounded set $S=\overline{\mathscr{B}}\left(x_{0}, r\right)$, where $r=\left\|x_{0}\right\|+L+F_{0}+V$ and define the operator $Q$ on $S$ into itself by (5.10). Then proceeding as in the Step IV of the proof of Theorem 5.1 it can be proved that $Q$ is a nonlinear $\mathscr{D}$-set-contraction with respect to the characteristic value $\omega_{0}$ with $\mathscr{D}$-function $\psi(r)=\frac{L r}{K+r}$, i.e., the inequality (5.18) holds on $E$. 
Next, we show that $Q$ is nonlinear $\mathscr{D}$-set-contraction with respect to the characteristic value $\delta_{a}$. Let $X$ be a bounded chain in $E$. Now, taking into account our assumptions, for arbitrarily fixed $t \in \mathbb{R}_{+}$and for $x \in X$ with $x \geq c$, we deduce the following estimate:

$$
\begin{aligned}
|(Q x)(t)-c| & \leq\left|f\left(t, x\left(\alpha_{1}(t)\right), x\left(\alpha_{2}(t)\right)\right)-f(t, c, c)\right|+a(t) \int_{0}^{\beta(t)} b(s) d s \\
& \leq \frac{L \max \left\{\left|x\left(\alpha_{1}(t)\right)-c\right|,\left|x\left(\alpha_{2}(t)\right)-c\right|\right\}}{K+\max \left\{\left|x\left(\alpha_{1}(t)\right)-c\right|,\left|x\left(\alpha_{2}(t)\right)-c\right|\right\}}+v(t) .
\end{aligned}
$$

From the above inequality it follows that

$$
|Q X(t)-c| \leq \frac{L \max \left\{\left|X\left(\alpha_{1}(t)\right)-c\right|,\left|X\left(\alpha_{2}(t)\right)-c\right|\right\}}{K+\max \left\{\left|X\left(\alpha_{1}(t)\right)-c\right|,\left|X\left(\alpha_{2}(t)\right)-c\right|\right\}}+v(t)
$$

for each $t \in \mathbb{R}_{+}$. Therefore, taking limit superior over $t \rightarrow \infty$, we obtain

$$
\begin{aligned}
\delta_{b}(Q X) & =\limsup _{t \rightarrow \infty}|Q(X(t))-c| \\
& \leq \frac{L \max \left\{\limsup _{t \rightarrow \infty}\left|X\left(\alpha_{1}(t)\right)-c\right|, \limsup _{t \rightarrow \infty}\left|X\left(\alpha_{2}(t)\right)-c\right|\right\}}{K+\max \left\{\limsup _{t \rightarrow \infty}\left|X\left(\alpha_{1}(t)\right)-c\right|, \limsup _{t \rightarrow \infty}\left|X\left(\alpha_{2}(t)\right)-c\right|\right\}} \\
& \leq \frac{L \limsup _{t \rightarrow \infty}|X(t)-c|}{K+\limsup _{t \rightarrow \infty}|X(t)-c|} \\
& =\frac{L \delta_{b}(X)}{K+\delta_{b}(X)} .
\end{aligned}
$$

Further, using the measure of noncompactness $\mu_{b}^{p}$ defined by the formula (5.3) and keeping in mind the estimates (5.18) and (5.20), we obtain

$$
\begin{aligned}
\mu_{b}^{p}(Q X) & =\max \left\{\omega_{0}(Q X), \delta_{b}(Q X)\right\} \\
& \leq \max \left\{\frac{L \omega_{0}(X)}{K+\omega_{0}(X)}, \frac{L \delta_{b}(X)}{K+\delta_{b}(X)}\right\} \\
& \leq \frac{L \max \left\{\omega_{0}(X), \delta_{b}(X)\right\}}{K+\max \left\{\omega_{0}(X), \delta_{b}(X)\right\}} . \\
& =\frac{L \mu_{b}^{p}(X)}{K+\mu_{b}^{p}(X)}
\end{aligned}
$$

for all chains $X$ in $S$. Since $L \leq K$, the operator $Q$ is a partially nonlinear $\mathscr{D}$-set-contraction on $S$ with $\mathscr{D}$-function $\psi(r)=\frac{L r}{K+r}$. This shows that $Q$ is a partially nonlinear $k$-set-contraction on $S$ with $k=L<1$. Again, by hypothesis $\left(\mathrm{H}_{5}\right)$, there exists an element $x_{0}=u \in S$ such that $x_{0} \leq Q x_{0}$, that is, $x_{0}$ is a lower solution of the FIE (5.8) defined on $\mathbb{R}_{+}$. The rest of the proof is similar to Theorem 5.1 and now we conclude from Remark 3.3 that the set $\mathscr{F}(Q)$ belongs 
to the family ker $\mu_{b}^{p}$. Now, taking into account the description of sets belonging to ker $\mu_{b}^{p}$ (given in Section 2) we deduce that the equation (5.8) has a solution $x^{*}$ and the sequence $\left\{x_{n}\right\}$ of successive iterations defined by (5.9) converges monotonically to $x^{*}$. Moreover, all comparable solutions of the equation (5.8) are uniformly locally ultimately asymptotically stable to the line $x(t)=c$ on $\mathbb{R}_{+}$. This completes the proof.

If $c=0$ in hypothesis $\left(\mathrm{H}_{7}\right)$, we obtain the following existence result concerning the asymptotic stability of the comparable solutions defined on $\mathbb{R}_{+}$.

Theorem 5.3. Assume that the hypotheses $\left(H_{1}\right)$ through $\left(H_{7}\right)$ hold with $c=0$. Then the functional FIE (5.8) has at least one solution $x^{*}$ in the space $B C\left(\mathbb{R}_{+}, \mathbb{R}\right)$ and the sequence $\left\{x_{n}\right\}$ of successive approximations defined by (5.9) converges monotonically to $x^{*}$. Moreover, the comparable solutions of the equation (5.8) are uniformly globally ultimately asymptotically stable to 0 defined on $\mathbb{R}_{+}$.

Remark 5.2. We remark that if a nonlinear hybrid integral equation (5.8) has more than one lower solution, then it may have a number of comparable lower solutions. Furthermore, the order relation $\leq$ in $C\left(\mathbb{R}_{+}, \mathbb{R}\right)$ is same as the order relation induced by the order cone

$$
\mathscr{K}=\left\{x \in C\left(\mathbb{R}_{+}, \mathbb{R}\right) \mid x(t) \geq 0 \text { for all } t \in \mathbb{R}_{+}\right\}
$$

in $C\left(\mathbb{R}_{+}, \mathbb{R}\right)$. Hence, the integral equation (5.8) has a number of comparable solutions defined on $\mathbb{R}_{+}$. As a result, under the given conditions of Theorem 5.1 all the comparable solutions of the nonlinear functional integral equation (5.8) are uniformly globally ultimately attractive on $\mathbb{R}_{+}$

Remark 5.3. The conclusion of Theorems 5.1, 5.2 and 5.3 remains true if we replace the hypothesis $\left(\mathrm{H}_{5}\right)$ with the following one:

$\left(\mathrm{H}_{5}^{\prime}\right)$ There exists an element $u \in C\left(\mathbb{R}_{+}, \mathbb{R}\right)$ such that

$$
u(t) \geq f(t, u(\alpha(t)))+\int_{t_{0}}^{\beta(t))} g(t, s, u(\gamma(s))) d s
$$

for all $t \in \mathbb{R}_{+}$.

The proof under this new hypothesis is similar to Theorem 5.1 and 5.2 and now, the desired conclusion follows by an application of Theorem 3.2.

Remark 5.4. The conclusion of Theorems 5.1 and 5.2 also remains true if we replace the hypothesis $\left(\mathrm{H}_{3}\right)$ with the following one:

$\left(\mathrm{H}_{3}^{\prime}\right)$ There exists a continuous and nondecreasing function $\phi: \mathbb{R}_{+} \rightarrow \mathbb{R}_{+}$such that

$$
0 \leq f(t, x)-f(t, y) \leq \phi(x-y)
$$

for all $t \in \mathbb{R}_{+}$and $x, y \in \mathbb{R}, x \geq y$, where $\phi(r)<r$ for $r>0$. 
In the following we give a numerical example to illustrate the abstract theory developed in this paper.

Example 5.1. Consider the linearly perturbed nonlinear hybrid fractional integral equation,

$$
x(t)=\tan ^{-1} x(2 t)+\int_{0}^{3 t} \frac{1}{t^{2}+1} g(s, x(s / 2)) d s
$$

for all $t \in \mathbb{R}_{+}$, where $g: \mathbb{R}_{+} \times \mathbb{R} \rightarrow \mathbb{R}$ is a function defined by

$$
g(t, x)=\left\{\begin{array}{cll}
1, & \text { if } & x \leq 0 \\
1+\frac{x}{x+1}, & \text { if } & x>0
\end{array}\right.
$$

We shall show that all the hypotheses of Theorem 3.1 are satisfied by the functions involved in FIE 5.21. Here, $\alpha(t)=2 t, \beta(t)=3 t$ and $\gamma(t)=t / 2$ and so, $\alpha, \beta, \gamma$ are continuous on $\mathbb{R}_{+}$into itself and $\alpha(t) \geq t$ for all $t \in \mathbb{R}_{+}$. Thus, hypothesis $\left(\mathrm{H}_{0}\right)$ is satisfied. Again, $f(t, x)=\tan ^{-1} x$ so that $f(t, x)$ is nondecreasing in $x$ for each $t \in \mathbb{R}_{+}$and continuous on $\mathbb{R}_{+} \times \mathbb{R}$. Also $f$ is bounded on $\mathbb{R}_{+} \times \mathbb{R}$ by the constant $\pi / 2$. The function $g(t, s, x)$ is given by $g(t, s, x)=\frac{1}{t^{2}+1} g(s, x)$. Next, $g(t, s, x)$ is defines a continuous function on $\mathbb{R}_{+} \times \mathbb{R}_{+} \times \mathbb{R}$ and nondecreasing function in $x$ for each $t, s \in \mathbb{R}_{+}$and so $\left(\mathrm{H}_{4}\right)$ holds. Moreover, $f(t, 0)=0$. So the hypotheses $\left(\mathrm{H}_{1}\right),\left(\mathrm{H}_{2}\right),\left(\mathrm{H}_{4}\right)$ and $\left(\mathrm{H}_{5}\right)$ are held.

Now, we show that $f$ is partially Lipschitz on $\mathbb{R}_{+} \times \mathbb{R}$. Let $x, y \in \mathbb{R}$ with $x \geq y$. Then,

$$
0 \leq f(t, x)-f(t, y)=\tan ^{-1} x-\tan ^{-1} y=\frac{1}{1+\xi^{2}}(x-y)
$$

for all $y<\xi<x$, and so hypothesis $\left(\mathrm{H}_{3}^{\prime}\right)$ is satisfied with the function $\phi$ defined by $\phi(r)=$ $\frac{r}{1+\xi^{2}}, 0<\xi<r$.

Furthermore, $|g(s, x)| \leq 2$ for all $s \in \mathbb{R}_{+}$and $x \in \mathbb{R}$. Therefore, we have

$$
v(t)=\int_{0}^{3 t} \frac{1}{t^{2}+1} \cdot 2 d s=\frac{6 t}{t^{2}+1} .
$$

Therefore,

$$
\lim _{t \rightarrow \infty} v(t)=\lim _{t \rightarrow \infty} \frac{6 t}{t^{2}+1}=0
$$

Finally, it is easy to prove that $u \equiv 0$ is a lower solution of the FIE (5.21) defined on $\mathbb{R}_{+}$and hence the hypothesis $\left(\mathrm{H}_{6}\right)$ is held. Thus all the conditions of Theorem 5.1 are satisfied and by a direct application, we conclude that the FIE (5.21) has a solution $x^{*}$ and the sequence $\left\{x_{n}\right\}$ defined by

$$
x_{n+1}(t)=\tan ^{-1} x_{n}(2 t)+\int_{0}^{3 t} \frac{1}{t^{2}+1} g\left(s, x_{n}(s / 2)\right) d s, t \in \mathbb{R}_{+} \text {, }
$$


converges monotonically to $x^{*}$, where $x_{0}=0$. Moreover, the comparable solutions of the FIE (5.21) are uniformly globally asymptotically ultimately attractive and stable to 0 defined on $\mathbb{R}_{+}$.

Remark 5.5. In this paper we have been able to weaken the Lipschitz condition to nonlinear one-sided or partial Lipschitz condition which otherwise is considered to be a very strong condition in the existence theory for nonlinear differential and integral equations. However, in this case we needed an additional assumption of monotonicity on the nonlinearities involved in the considered integral equation in order to guarantee the required characterization of attractivity of the comparable solutions defined on the unbounded intervals of real line.

Remark 5.6. The existence theorems proved in Section 5 may be extended with appropriate modifications to the generalized nonlinear hybrid functional integral equation

$$
\left.x(t)=f\left(t, x\left(\alpha_{1}(t)\right), \ldots, x\left(\alpha_{n}(t)\right)\right)+\int_{0}^{\beta(t)} g\left(t, s, x\left(\gamma_{1}(s)\right), \ldots, \gamma_{n}(s)\right)\right) d s
$$

for all $t \in \mathbb{R}_{+}$, where $\alpha_{i}, \beta, \gamma_{i}: \mathbb{R}_{+} \rightarrow \mathbb{R}_{+}, i=1,2, \ldots, n, f: \mathbb{R}_{+} \times \mathbb{R}^{n} \rightarrow \mathbb{R}$, and $g: \mathbb{R}_{+} \times \mathbb{R}_{+} \times \mathbb{R}^{n} \rightarrow \mathbb{R}$ are continuous functions.

\section{Conclusion}

Observe that the fixed point theorems of this paper may be applied to nonlinear hybrid fractional integral equations for proving the existence results, however unlike existence theorem for nonlinear hybrid integral equations discussed in Dhage [8] we do not require the assumption that $E$ to be a lattice. Again the continuity of the functions $f(t, x)$ and $g(t, s, x)$ in the variable $x$ means that they are partially continuous on $\mathbb{R}$ since $\mathbb{R}$ is a totally ordered set, and therefore, the corresponding operators defined in the proof of above theorem are partially continuous on the domains of their definition which is contrary to the case considered in Banas and Dhage [3] and Banas and Rzepka [4]. Moreover, we have proved the existence and attractivity of comparable solutions under weak one sided or partial Lipschitz condition, however, in this case we assumed the nonlinearities in question are monotonic in unknown variable together with the existence of a lower solution of the considered nonlinear integral equations. The advantage of the present approach over previous ones is that we have been able to develop an algorithm for the solutions of the considered integral equations which otherwise is not possible via classical approach of measure of noncompactness treated in Banas and Goebel [2]. Another interesting feature of our work is that we generally need the uniqueness of the solution for predicting the behavior of the dynamic systems related to the considered nonlinear fractional integral equation, however with the present approach it possible for us to discuss the qualitative behavior of the systems even though there exist a number of 
solutions of the dynamic system in question. Finally, while concluding this paper, we mention that the above existence theorem may be proved by using Theorem 4.2 under weaker Carathéodory condition than continuity of the nonlinearities with appropriate modifications and some of the results in above mentioned direction will be reported elsewhere.

\section{References}

[1] J. Appell, Measures of noncompactness, condensing operators and fixed points : An application-oriented survey, Fixed Point Theory, 6 (2005), 157-229.

[2] J. Banas, K. Goebel, Measures of Noncompactness in Banach Space, in: Lecture Notes in Pure and Applied Mathematics, Vol. 60, Dekker, New York, 1980.

[3] J. Banas and B. C. Dhage, Global asymptotic stability of solutions of a functional integral equations, Nonlinear Analysis, 69 (2008), 1945-1952

[4] J. Banas and B. Rzepka, An application of a measure noncompactness in the study of asymptotic stability, Appl. Math. Lett., 16 (2003), 1-6.

[5] S. Carl and S. Hekkilä, Fixed Point Theory in Ordered Sets and Applications, Springer, 2011.

[6] B. C. Dhage, Fixed point theorems in ordered Banach algebras and applications, PanAmer. Math. J., 9(4) (1999), 93-102.

[7] B. C. Dhage, Fixed point theorems in ordered Banach algebras and applications, PanAmer. Math. J. 9(4) (1999), 93-102.

[8] B. C. Dhage, Hybrid fixed point theory in partially ordered normed linear spaces and applications to fractional integral equations, Differ. Equ Appl., 5 (2013), 155-184.

[9] B. C. Dhage, Global attractivity results for comparable solutions of nonlinear hybrid fractional integral equations, Differ. Equ Appl. 6 (2014), 165-186.

[10] B. C. Dhage and S. B. Dhage, Approximating solutions of nonlinear pbups of hybrid differential equations via hybrid fixed point theory, Indian J. Math., 56 (2014) (In press).

[11] B. C. Dhage and S. B. Dhage, Global attractivity and stability results for comparable solutions of nonlinear fractional integral equations, Nonlinear Studies, 21 (2014), 255-268.

[12] B. C. Dhage, S. B. Dhage and D. V. Mule, Local attractivity and stability results for hybrid functional nonlinear fractional integral equations, Nonlinear Funct. Anal. \& Appl., 19, (2014), 415-433.

[13] B. C. Dhage, S. B. Dhage and S. K. Ntouyas, Approximating solutions of nonlinear hybrid differential equations, Appl. Math. Lett., 34 (2014), 76-80.

[14] B. C. Dhage, V. Lakshmikatham, On global existence and attrctivity results for nonlinear functional integral equations, Nonlinear Analysis, 72 (2010), 2219-2227.

[15] I. T. Gokhberg, I. S. Goldstein and A. S. Markus, Investigation of some properties of bounded linear operators in connection with their q-norm, Uch zap Kishinevsk In a 29 (1957), 29-36.

[16] S. Heikkilä and V. Lakshmikantham, Monotone Iterative Techniques for Discontinuous Nonlinear Differential Equations, Marcel Dekker inc., New York 1994.

[17] M. A. Krasnoselskii, Topological Methods in the Theory of Nonlinear Integral Equations, Pergamon Press 1964.

[18] C. Kuratowskii, Sur les espaces complete, Fund. Math., 15 (1930), 301-309.

[19] J. J. Nieto and R. Rodriguez-Lopez, Contractive mappings theorems in partially ordered sets and applications to ordinary differential equations, Order, 22 (2005), 223-239.

[20] A. C. M. Ran and M. C. R. Reurings, A fixed point theorem in partially ordered sets and some applications to matrix equations, Proc. Amer. Math. Soc., 132 (2003), 1435-1443.

Kasubai, Gurukul Colony, Ahmedpur-413 515, Dist: Latur, Maharashtra, India.

E-mail: bcdhage@gmail.com 